\title{
Hyperbolic and Elliptic Transport Barriers in Three-Dimensional Unsteady Flows
}

\author{
Daniel Blazevski, George Haller \\ Institute for Mechanical Systems, Department of Mechanical and Process Engineering, \\ ETH Zurich, Tannenstrasse 3, Zurich, Switzerland
}

\begin{abstract}
We develop a general theory of transport barriers for three-dimensional unsteady flows with arbitrary time-dependence. The barriers are obtained as two-dimensional Lagrangian Coherent Structures (LCSs) that create locally maximal deformation. Along hyperbolic LCSs, this deformation is induced by locally maximal normal repulsion or attraction. Along shear LCSs, the deformation is created by locally maximal tangential shear. Hyperbolic LCSs, therefore, play the role of generalized stable and unstable manifolds, while closed shear LCSs (elliptic LCSs) act as generalized KAM tori or KAM-type cylinders. All these barriers can be computed from our theory as explicitly parametrized surfaces. We illustrate our results by visualizing two-dimensional hyperbolic and elliptic barriers in steady and unsteady versions of the $\mathrm{ABC}$ flow.
\end{abstract}

\section{Introduction}

Detecting transport barriers is important in a number of areas, including geophysical flows [29], plasma fusion [25], reactive flows [24], and molecular dynamics [28]. For steady and temporally periodic flow models in these areas, classical dynamical systems theory identifies key invariant manifolds acting as phase space barriers [22]. Even in this well-understood setting, however, only specific examples of de facto barriers have been identified. Indeed, even for steady flows, no general approach to defining and locating multi-dimensional transport barriers has been available. A commonly used informal definition of barriers as surfaces with zero transverse flux is easily seen to be inadequate. Indeed, any randomly chosen surface of trajectories (material surface) admits zero normal flux [16].

A number of heuristic flow diagnostics have nevertheless been employed to infer transport barriers indirectly, mostly targeting two-dimensional flows (see [4] and [23] for reviews). These diagnostics can be highly effective for simple flows, especially if one tunes their threshold parameters to match expectations. However, when it comes to discovering more complex flows, the lack of an exact mathematical foundation renders these approaches problematic, manifested by frame-dependence, false positives and false negatives $[2,18,14]$.

For exploration, decision making and forecasting, one would ideally require a general approach with solid mathematical foundations. Such a general approach, the geodesic theory of transport barriers, has recently emerged for two-dimensional unsteady flows $[16,6,17]$. Specifically, Haller and Beron-Vera [16] constructs transport barriers as curves most closely shadowed by least-stretching geodesics of the Cauchy-Green strain tensor derived from the flow map. As a further improvement, F. J. Beron-Vera and Haller [6], Haller and Beron-Vera [17] constructs transport barriers as distinguished geodesics of the Green-Lagrange strain tensors derived from the flow map. The objective of the present work is to extend these ideas to unsteady flows in three dimensions.

We are unaware of other approaches that would directly target transport barrier surfaces in multi-dimensional unsteady flows. Scalar fields associated with the flow map such as the finite-time Lyapunov exponents (FTLE) and finite-size Lyapunov exponents have been used as indicators of 
hyperbolic coherent structures $[13,21,3,27,11,26]$. Under certain conditions, select ridges of these fields can be rigorously related to repelling and attracting transport barriers $[15,8,7,19]$.

Related methods exist for multi-dimensional coherence detection, including an ergodicity-based approach to visualizing elliptic structures in steady and temporally periodic flows [5], as well as a probabilistic approach to locating almost invariant sets in phase space [10]. Both methods seek coherent domains (ergodic components or almost invariant sets) via a modal truncation of an infinitedimensional operator (the Koopman or the Peron-Frobenius operator, respectively). This process then yields scalar fields whose topology is expected to reveal coherent sets. Specifically, in [5], lowindex eigenfunctions are proposed as indicators of dynamically distinct regions of the phase space. In [10], the left and right eigenfunctions of the second largest singular value of the Peron-Frobenius operator are thresholded to maximize the coherence ratio of pair of sets.

Our focus here is a direct variational construction of transport barriers as parametrized surfaces. Instead of starting with a particular mathematical quantity and arguing for its relevance in barrier detection, we start with a physically motivated question: What objective property makes transport barriers observable in physical and numerical experiments?

We put forward the same answer that has been well-tested in two-dimensional flows. Specifically, for a time-evolving surface $\mathcal{M}(t)$ to be an observed transport barrier, the following two properties should hold:

T1 $\mathcal{M}(t)$ must be a material surface, i.e., a two-dimensional invariant manifold in the extended phase space of positions and time. This implies that barriers locally divide the phase space and have zero flux between their two sides.

T2 $\mathcal{M}(t)$ must impose locally extreme deformation on nearby sets of initial conditions. This is achieved either by locally maximal normal repulsion or attraction (hyperbolic barrier), or locally maximal tangential shear (shear barrier).

Properties T1-T2 provide an extension of the concept of a multi-dimensional Lagrangian Coherent Structure (LCSs) from the purely hyperbolic case treated in [15] to the general case. Solving the extremum problem described in T2 leads to vector fields of admissible unit normals for hyperbolic and shear barriers. It turns out that surfaces orthogonal to these admissible normal fields can only exist at locations where the helicity of these normal fields vanishes. Strain- and shear-helicity generically vanish on computable two-dimensional surfaces; these zero sets necessarily contain the transport barriers we seek.

The intersection of transport barriers with select two-dimensional reference surfaces turns out to satisfy ordinary differential equations (strain and shear ODEs). These ODEs can be solved numerically, yielding parametrized reduced strainlines and reduced shearlines on the reference surfaces. Open reduced shearlines of zero helicity signal generalized jets (parabolic barriers), while closed reduced shearlines mark invariant tubes or invariant tori (elliptic barriers). Extracting such parametrized curves over a parametrized family of reference surfaces leads to explicitly parametrized two-dimensional transport barriers.

This construction applies to any three-dimensional flow with general time-dependence, and uncovers key barriers that shape tracer patterns over a finite time of observation. This time can be arbitrarily short or long: our approach, by construction, will locate barriers that best explain tracer patterns developing over the observational period chosen. Over longer time intervals, the same approach yields increasingly accurate approximations for classic hyperbolic and elliptic invariant manifolds, should those exist in the given flow.

We first illustrate these results on the steady and time-periodic ABC flows, which have welldefined steady and time-periodic transport barriers given by invariant manifolds. Even in these flows, we obtain new, explicit barrier surfaces that were previously only inferred from numerical images. Next, we consider a chaotically forced version of the ABC flow over a finite time interval. For this flow, transport barriers can only be constructed as temporally aperiodic material surfaces in the extended phase space. Remarkably, we obtain that select hyperbolic barriers and torus-type 
shear barriers continue to exist even in this fully aperiodic setting. The latter tori tori deform aperiodically in time, yet continue to provide sharp boundaries for coherent Lagrangian vortices. Indeed, they exhibit minimal deformation while nearby material elements in their exteriors stretch exponentially.

\section{Set-up and notation}

Consider the dynamical system

$$
\dot{x}=v(x, t), \quad x \in D \subset \mathbb{R}^{3}, \quad t \in\left[t_{0}, t_{0}+T\right],
$$

with a smooth vector field $v(x, t)$ defined over a time interval of length $T$, for locations $x$ in a compact set $D$. We assume a finite time time interval in (1) since data sets obtained from physical measurements or numerical methods are only known over such intervals. Moreover, coherent structures of physical interest are typically transient in nature (eddies, hurricanes, etc).

A trajectory of (1) starting from $x_{0}$ at time $t_{0}$ is denoted by $x\left(t ; t_{0}, x_{0}\right)$. The flow map of $(1)$ is then defined as

$$
F_{t_{0}}^{t}\left(x_{0}\right): x_{0} \mapsto x\left(t ; t_{0}, x_{0}\right),
$$

which is as smooth in $x_{0}$ as the function $v(x, t)$ in $x$.

Assuming that $v$ is of $C^{r}$ with $r \geq 1$, the flow gradient $\nabla F_{t_{0}}^{t}\left(x_{0}\right)$ can be computed. This linear mapping advects small initial perturbations $\xi_{0}$ to $x_{0}$ along the trajectory $x\left(t, t_{0}, x_{0}\right)$ to the evolved perturbation $\xi_{t}=\nabla F_{t_{0}}^{t}\left(x_{0}\right) \xi_{0}$. Note that

$$
\left|\xi_{t}\right|^{2}=\left\langle\xi_{0}, C_{t_{0}}^{t}\left(x_{0}\right) \xi_{0}\right\rangle
$$

where $C_{t_{0}}^{t}\left(x_{0}\right):=\left[\nabla F_{t_{0}}^{t}\left(x_{0}\right)\right]^{*} \nabla F_{t_{0}}^{t}\left(x_{0}\right)$ denotes the Cauchy-Green strain tensor, and $\left.<\cdot, \cdot\right\rangle$ is the classic Euclidean inner product.

We will be interested in stationary values of total perturbation growth (3) over the time interval $\left[t_{0}, t_{0}+T\right]$. These values are precisely the eigenvalues of the symmetric, positive definite matrix $C_{t_{0}}^{t_{0}+T}\left(x_{0}\right)$. The eigenvalues $\lambda_{i}\left(x_{0}\right)$ and their corresponding orthonormal eigenvectors $\xi_{i}\left(x_{0}\right)$ satisfy

$$
C_{t_{0}}^{t_{0}+T}\left(x_{0}\right) \xi_{i}\left(x_{0}\right)=\lambda_{i}\left(x_{0}\right) \xi_{i}\left(x_{0}\right), \quad\left|\xi_{i}\left(x_{0}\right)\right|=1, \quad i=1,2,3 .
$$

From now on, we restrict our discussion to an open set $U$ of initial conditions where the eigenvalues of $C_{t_{0}}^{t_{0}+T}\left(x_{0}\right)$ are disjoint:

$$
U=\left\{x_{0} \in D: 0<\lambda_{1}\left(x_{0}\right)<\lambda_{2}\left(x_{0}\right)<\lambda_{3}\left(x_{0}\right)\right\} .
$$

\section{Three-dimensional transport barriers}

Here we give a formal definition of transport barriers building on the properties T1-T2 described in the Introduction. According to T1, a time-dependent transport barrier $\mathcal{M}(t)$ must be a material surface, i.e., an invariant manifold in the extended phase space of the variables $(x, t)$. This necessarily implies

$$
\mathcal{M}(t)=F_{t_{0}}^{t}\left(\mathcal{M}\left(t_{0}\right)\right),
$$

for any time $t \in\left[t_{0}, t_{0}+T\right]$. As long as $\mathcal{M}\left(t_{0}\right)$ is a smooth surface, so is the surface $\mathcal{M}(t)$ for any fixed time $t$. The family $\mathcal{M}(t)$ is equally smooth in $t$ by our smoothness assumption on (1).

At an initial point $x_{0} \in \mathcal{M}\left(t_{0}\right)$, let $n_{0}$ denote a unit normal to $\mathcal{M}\left(t_{0}\right)$. Then, as discussed in [15], a smoothly varying unit normal to $\mathcal{M}(t)$ along the trajectory $x\left(t, t_{0}, x_{0}\right)$ is given by

$$
n_{t}\left(x_{0}\right)=\frac{\left[\nabla F_{t}^{t_{0}}\left(x_{0}\right)\right]^{*} n_{0}}{\left|\left[\nabla F_{t}^{t^{0}}\left(x_{0}\right)\right]^{*} n_{0}\right|} .
$$



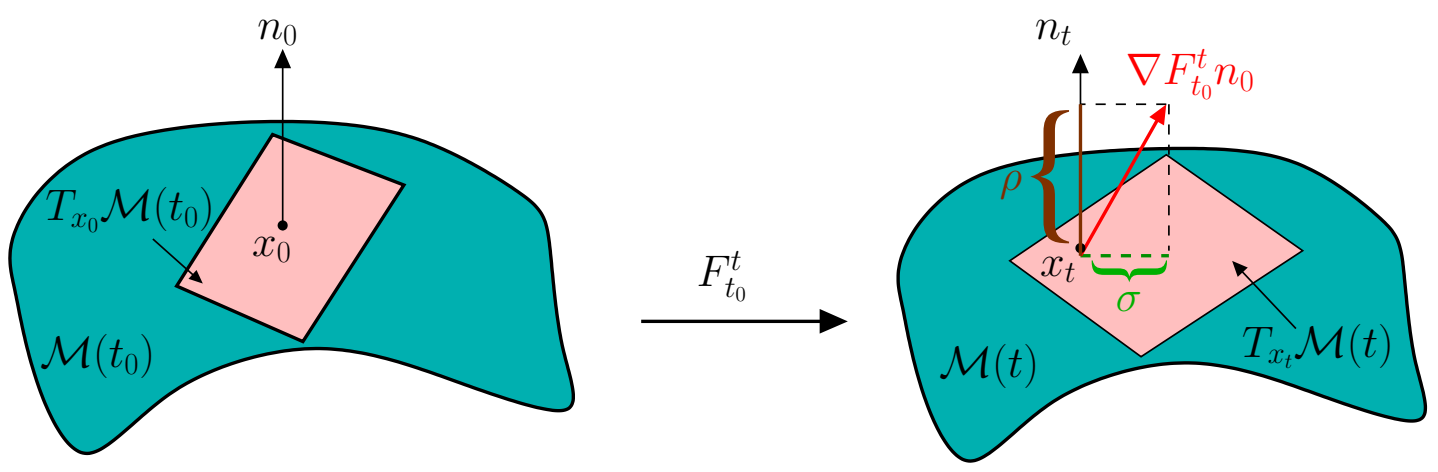

Figure 1: The definition of normal repulsion and tangential shear along a material surface.

For any initial point $x_{0} \in \mathcal{M}\left(t_{0}\right)$ and initial unit normal $n_{0}$ to $\mathcal{M}\left(t_{0}\right)$ at $x_{0}$, we define the normal repulsion $\rho\left(x_{0}, n_{0}\right)$ of $\mathcal{M}(t)$ along the trajectory $x\left(t ; t_{0}, x_{0}\right)$ as the normal component of the growth of $n_{0}$ under the linearized flow between times $t_{0}$ and $t_{0}+T$ [15]. Specifically, we have

$$
\rho\left(x_{0}, n_{0}\right)=\left\langle n_{t_{0}+T}\left(x_{0}\right), \nabla F_{t_{0}}^{t_{0}+T}\left(x_{0}\right) n_{0}\right\rangle,
$$

with the geometry illustrated in Fig. 1. Similarly, we define the tangential shear $\sigma\left(x_{0}, n_{0}\right)$ as the tangential component of the growth of $n_{0}$ under the linearized flow along the trajectory $x\left(t ; t_{0}, x_{0}\right)$ between times $t_{0}$ and $t_{0}+T[16]$. Specifically, we have

$$
\sigma\left(x_{0}, n_{0}\right)=\left|\nabla F_{t_{0}}^{t_{0}+T}\left(x_{0}\right) n_{0}-\left\langle n_{t_{0}+T}\left(x_{0}\right), \nabla F_{t_{0}}^{t_{0}+T}\left(x_{0}\right) n_{0}\right\rangle n_{t_{0}+T}\left(x_{0}\right)\right|
$$

with the geometry shown in Fig. 1.

We seek transport barriers as material surfaces that maximize normal repulsion or tangential shear with respect to perturbations to their tangent spaces. We do not insist on this maximizing property under all perturbations to the material surface: we only consider perturbations to their tangent spaces. This is because we seek a well-defined local directionality for the transport barrier, while in principle allowing for it to have a finite thickness. In other words, the barrier may a priori be a thin set of nearby, parallel surfaces.

\section{Definition 1.}

(i) A material surface $\mathcal{M}(t) \subset \mathbb{R}^{3}$ is called a repelling hyperbolic LCS over the interval $\left[t_{0}, t_{0}+T\right]$, if for any point $x_{0} \in \mathcal{M}\left(t_{0}\right)$ and for any other material surface $\hat{\mathcal{M}}\left(t_{0}\right)$ with $x_{0} \in \hat{\mathcal{M}}\left(t_{0}\right)$ and with unit normal $\hat{n}_{0} \nVdash n_{0}$ at $x_{0}$, we have

$$
\rho\left(x_{0}, \hat{n}_{0}\right)<\rho\left(x_{0}, n_{0}\right), \quad \rho\left(x_{0}, n_{0}\right)>1 .
$$

(ii) A material surface $\mathcal{M}(t) \subset \mathbb{R}^{3}$ is called an attracting hyperbolic $L C S$ over the interval $\left[t_{0}, t_{0}+T\right]$, if for any point $x_{0} \in \mathcal{M}\left(t_{0}\right)$ and for any other material surface $\hat{\mathcal{M}}\left(t_{0}\right)$ with $x_{0} \in \hat{\mathcal{M}}\left(t_{0}\right)$ and with unit normal $\hat{n}_{0} \nVdash n_{0}$ at $x_{0}$, we have

$$
\rho\left(x_{0}, \hat{n}_{0}\right)>\rho\left(x_{0}, n_{0}\right), \quad \rho\left(x_{0}, n_{0}\right)<1 .
$$

(iii) A material surface $\mathcal{M}(t) \subset \mathbb{R}^{3}$ is called a shear $L C S$ over the interval $\left[t_{0}, t_{0}+T\right]$, if for any point $x_{0} \in \mathcal{M}\left(t_{0}\right)$ and for for any other material surface $\hat{\mathcal{M}}\left(t_{0}\right)$ with $x_{0} \in \hat{\mathcal{M}}\left(t_{0}\right)$ and with unit normal $\hat{n}_{0} \nVdash n_{0}$ at $x_{0}$, we have

$$
\sigma\left(x_{0}, \hat{n}_{0}\right) \leq \sigma\left(x_{0}, n_{0}\right),
$$

with $\hat{n}_{0}$ denoting a unit normal to $\hat{\mathcal{M}}\left(t_{0}\right)$ at the point $x_{0}$. 
(iv) A material surface $\mathcal{M}(t) \subset \mathbb{R}^{3}$ is called a transport barrier over the interval $\left[t_{0}, t_{0}+T\right]$, if it is either a hyperbolic or a shear LCS over $\left[t_{0}, t\right]$.

Remark 1. By (i) of Definition 1, hyperbolic LCS exhibit strictly maximal repulsion relative to all perturbations to their tangent spaces. This is to guarantee that any potential stretching in directions tangent to the LCS is smaller than in its normal direction. This condition guarantees both the observability and the robustness of a repelling hyperbolic barrier [15]. The same observation applies to attracting hyperbolic barriers in backward time.

Remark 2. By (ii) of Definition 1, the shear $\sigma\left(x_{0}, n_{0}\right)$ along a shear barrier $\mathcal{M}\left(t_{0}\right)$ is not required to be strictly maximal among all possible perturbations to the tangent space of $\mathcal{M}\left(t_{0}\right)$. As it turns out below, there are always two choices of the normal $n_{0}$ yielding the exact same, locally largest value of $\sigma\left(x_{0}, n_{0}\right)$ at any point $x_{0}$. Shear barriers, therefore, exhibit strictly maximal shear only with respect to small enough perturbations of their normals. There will always exist a unique, finite perturbation to their normal yielding exactly the same locally maximal shear at $x_{0}$. The two shear extrema at $x_{0}$ represent maximal shear with two different signs, which is disguised by the absolute value appearing in the definition of $\sigma\left(x_{0}, n_{0}\right)$.

The following theorem shows that for a material surface $\mathcal{M}(t)$ to be a transport barrier over $\left[t_{0}, t_{0}+T\right]$, its initial position must be orthogonal to a direction characterizing maximal repulsion or maximal shear. We use the notation $T_{x_{0}} \mathcal{M}\left(t_{0}\right)$ for the tangent space of $\mathcal{M}\left(t_{0}\right)$ at a point $x_{0}$.

Theorem 1. [Existence of transport barriers] Let $\mathcal{M}(t) \subset \mathbb{R}^{3}$ be a material surface over the time interval $\left[t_{0}, t_{0}+T\right]$. Then

(i) $\mathcal{M}(t)$ is a repelling hyperbolic LCS if and only if $\mathcal{M}\left(t_{0}\right) \subset U$ and $\xi_{3}\left(x_{0}\right) \perp T_{x_{0}} \mathcal{M}\left(t_{0}\right)$ holds for all $x_{0} \in \mathcal{M}\left(t_{0}\right)$.

(ii) $\mathcal{M}(t)$ is an attracting hyperbolic LCS if and only if $\mathcal{M}\left(t_{0}\right) \subset U$, and $\xi_{1}\left(x_{0}\right) \perp T_{x_{0}} \mathcal{M}\left(t_{0}\right)$ holds for all $x_{0} \in \mathcal{M}\left(t_{0}\right)$.

(iii) $\mathcal{M}(t)$ is a shear LCS if and only if $\mathcal{M}\left(t_{0}\right) \subset U$, and $n_{ \pm}\left(x_{0}\right) \perp T_{x_{0}} \mathcal{M}\left(t_{0}\right)$ holds for all $x_{0} \in \mathcal{M}\left(t_{0}\right)$ for one choice of the sign \pm in the vector field

$$
n_{ \pm}\left(x_{0}\right)=\sqrt{\frac{\sqrt{\lambda_{1}\left(x_{0}\right)}}{\sqrt{\lambda_{1}\left(x_{0}\right)}+\sqrt{\lambda_{n}\left(x_{0}\right)}}} \xi_{1}\left(x_{0}\right) \pm \sqrt{\frac{\sqrt{\lambda_{3}\left(x_{0}\right)}}{\sqrt{\lambda_{1}\left(x_{0}\right)}+\sqrt{\lambda_{3}\left(x_{0}\right)}}} \xi_{3}\left(x_{0}\right) .
$$

Proof. See Appendix C.

Remark 3. The above necessary conditions for hyperbolic LCS have previously been obtained from slightly different considerations, along with examples illustrating their meaning in $[15,7,9]$. For an explicit example of how shear LCS can be found in three-dimensional, unsteady parallel shear flows, we refer to Appendix A.

Remark 4. Unlike in the two-dimensional case [16], the shear LCSs obtained in (iii) of Theorem 1 generally do not preserve their surface area under an incompressible flow map $F_{t_{0}}^{t_{0}+T}$, even though they still preserve their enclosed volume (cf. Appendix B). This enables their use in detecting material footprints of commonly observed toroidal vortices, such as growing smoke rings.

Remark 5. A related recent paper [12] shows how quasi-invariant hyperbolic LCS can be used to compute a specific family of hyperbolic barriers (normally hyperbolic invariant manifolds) in steady flows of arbitrary dimension. 
Theorem 1 requires the initial position $\mathcal{M}\left(t_{0}\right)$ of a transport barrier to be orthogonal to $\xi_{3}$ (hyperbolic barrier) or to $n_{ \pm}$(shear barrier). In general, if a two-dimensional surface is orthogonal to a three-dimensional vector field $\rho(x)$, then any local parametrization $p\left(s_{1}, s_{2}\right): U \subset \mathbb{R}^{2} \rightarrow \mathbb{R}^{3}$ of the surface must satisfy the first-order quasi-linear system of PDEs

$$
\begin{aligned}
& \rho_{1}(p) \partial_{s_{1}} p_{1}+\rho_{2}(p) \partial_{s_{1}} p_{2}+\rho_{3}(p) \partial_{s_{1}} p_{3}=0, \\
& \rho_{1}(p) \partial_{s_{2}} p_{1}+\rho_{2}(p) \partial_{s_{2}} p_{2}+\rho_{3}(p) \partial_{s_{2}} p_{3}=0 .
\end{aligned}
$$

This system of PDEs will only have a smooth solution through a given point $x_{0}$ if this point is contained in a transport barrier. To locate such barrier points, we now give computable necessary conditions for transport barrier locations. In stating these conditions, we will use the helicity $H_{\rho}(x)$ of a three-dimensional vector field $\rho(x)$, defined as

$$
H_{\rho}(x)=\langle\nabla \times \rho(x), \rho(x)\rangle,
$$

with $\times$ denoting the cross product.

Theorem 2. [Necessary condition for transport barriers] Let $\mathcal{M}(t) \subset \mathbb{R}^{3}$ be a material surface over the time interval $\left[t_{0}, t_{0}+T\right]$.

(i) Suppose that $\mathcal{M}(t)$ is a repelling hyperbolic LCS. Then at all points $x_{0} \in \mathcal{M}\left(t_{0}\right)$, we must have

$$
H_{\xi_{3}}\left(x_{0}\right)=0 .
$$

(ii) Suppose that $\mathcal{M}(t)$ is an attracting hyperbolic LCS. Then at all points $x_{0} \in \mathcal{M}\left(t_{0}\right)$, we must have

$$
H_{\xi_{1}}\left(x_{0}\right)=0 .
$$

(iii) Suppose that $\mathcal{M}(t)$ is a shear LCS. Consider the two vector fields

$$
n_{ \pm}\left(x_{0}\right)=\sqrt{\frac{\sqrt{\lambda_{1}\left(x_{0}\right)}}{\sqrt{\lambda_{1}\left(x_{0}\right)}+\sqrt{\lambda_{3}\left(x_{0}\right)}}} \xi_{1}\left(x_{0}\right) \pm \sqrt{\frac{\sqrt{\lambda_{3}\left(x_{0}\right)}}{\sqrt{\lambda_{1}\left(x_{0}\right)}+\sqrt{\lambda_{3}\left(x_{0}\right)}}} \xi_{3}\left(x_{0}\right) .
$$

Then at all points $x_{0} \in \mathcal{M}\left(t_{0}\right)$, we must have

$$
H_{n_{ \pm}}\left(x_{0}\right)=0
$$

for one choice of the sign in \pm .

Proof. See Appendix D.

Remark 6 . The problem of finding surfaces orthogonal to vector fields is locally equivalent to finding surfaces tangent to two smooth vector fields. For the existence of such tangent surfaces, the Frobenius Integrability Theorem provides a necessary condition. This can be shown equivalent to the zero helicity conditions described above (cf. Appendix D).

Theorem 3 provides specific scalar equations of the form (11) and (13) that a transport barrier $\mathcal{M}\left(t_{0}\right)$ must satisfy. Rather than solving these equations numerically, we locate the intersection curves of all potential transport barriers with a family of two-dimensional reference surfaces. Out of all these intersection curves, we then select the ones on which the appropriate helicity condition in Theorem 2 vanishes. This leads to the following result:

Theorem 3. [Necessary condition for intersections of transport barriers with reference surfaces] Let $\mathcal{M}(t) \subset \mathbb{R}^{3}$ be a material surface over the time interval $\left[t_{0}, t_{0}+T\right]$. Also, let $\Pi\left(s_{1}\right)$ denote a smooth, one-parameter family of two-dimensional orientable surfaces with smooth normal vector fields $n_{\Pi\left(s_{1}\right)}(x)$. 
(i) Suppose that $\mathcal{M}\left(t_{0}\right)$ is a repelling hyperbolic LCS. Then the intersection curve $\gamma_{s_{1}}=\Pi\left(s_{1}\right) \cap$ $\mathcal{M}\left(t_{0}\right)$ is a trajectory of a two-dimensional differential equation on $\Pi\left(s_{1}\right)$, given by

$$
\gamma_{s_{1}}^{\prime}\left(s_{2}\right)=n_{\Pi\left(s_{1}\right)}\left(\gamma_{s_{1}}\left(s_{2}\right)\right) \times \xi_{3}\left(\gamma_{s_{1}}\left(s_{2}\right)\right) .
$$

This trajectory must also satisfy the condition

$$
H_{\xi_{3}}\left(\gamma_{s_{1}}\left(s_{2}\right)\right)=0
$$

(ii) Suppose that $\mathcal{M}\left(t_{0}\right)$ is an attracting hyperbolic LCS. Then the intersection curve $\gamma_{s_{1}}=\Pi\left(s_{1}\right) \cap$ $\mathcal{M}\left(t_{0}\right)$ is a trajectory of a two-dimensional differential equation on $\Pi\left(s_{1}\right)$, given by

$$
\gamma_{s_{1}}^{\prime}\left(s_{2}\right)=n_{\Pi\left(s_{1}\right)}\left(\gamma_{s_{1}}\left(s_{2}\right)\right) \times \xi_{1}\left(\gamma_{s_{1}}\left(s_{2}\right)\right) .
$$

This trajectory must also satisfy the condition

$$
H_{\xi_{1}}\left(\gamma_{s_{1}}\left(s_{2}\right)\right)=0
$$

(iii) Suppose that $\mathcal{M}\left(t_{0}\right)$ is a shear LCS. Then the intersection curve $\gamma_{s_{1}}=\Pi\left(s_{1}\right) \cap \mathcal{M}\left(t_{0}\right)$ is a trajectory of a two-dimensional differential equation on $\Pi\left(s_{1}\right)$, given by

$$
\gamma_{s_{1}}^{\prime}\left(s_{2}\right)=n_{\Pi\left(s_{1}\right)}\left(\gamma_{s_{1}}\left(s_{2}\right)\right) \times n_{ \pm}\left(\gamma_{s_{1}}\left(s_{2}\right)\right),
$$

for some choice of the sign in \pm . This trajectory must also satisfy the condition

$$
H_{n_{ \pm}}\left(\gamma_{s_{1}}\left(s_{2}\right)\right)=0
$$

with the same choice of the sign. If the trajectory $\gamma_{s_{1}}\left(s_{2}\right)$ is a closed orbit, then $\mathcal{M}(t)$ is an elliptic barrier.

Proof. See Appendix E.

Remark 7 . Theorem 3 yields a local parametrization $p\left(s_{1}, s_{2}\right)=\gamma_{s_{1}}\left(s_{2}\right)$ for transport barriers in the form of parametrized families of smooth curves $\gamma_{s_{1}}\left(s_{2}\right)$.

\section{Computation of transport barriers}

Theorem 3 provides a practical algorithm for the computation of transport barriers as different types of LCSs in three-dimensional flows. The barriers can be reconstructed from their intersections with a family of orientable hypersurfaces.

In the simplest case, these hypersurfaces are just two-dimensional planes. For this case, we summarize below the extraction of hyperbolic LCS (generalized stable and unstable manifolds) and elliptic LCS (invariant cylinders and generalized KAM tori). Further hints on the numerical implementation of these algorithmic steps can be found in Appendix D.

\subsection{Algorithm for hyperbolic LCSs}

H1 Compute the Cauchy-Green strain tensor $C_{t_{0}}^{t_{0}+T}$ and its dominant eigenvector $\xi_{3}$ on a twodimensional grid $\mathcal{G}_{0}$ defined on the reference plane $\Pi\left(s_{1}\right)$

H2 Pick a sparser grid $\mathcal{G}_{1}$ of initial conditions. Obtain reduced strainlines $\gamma_{s_{1}}\left(s_{2}\right)$ as trajectories of the ODE (14), starting from points of $\mathcal{G}_{1}$ satisfying $\left|H_{\xi_{3}}\right|=\left|\left\langle\nabla \times \xi_{3}, \xi_{3}\right\rangle\right|<\epsilon_{0}$ for some threshold parameter $\epsilon_{0}$.

H3 Integrate such reduced strainlines as long as the running average of $\left|H_{\xi_{3}}\right|$ stays below $\epsilon_{0}$. 
H4 Filter the reduced strainline segments so obtained to find the ones that approximate the zero sets of $H_{\xi_{3}}$ most closely. Specifically, if the Hausdorff distance

$$
d\left(\gamma_{s_{1}}, \tilde{\gamma}_{s_{1}}\right)=\max _{x \in \gamma_{1}}\left(\min _{y \in \tilde{\gamma}_{s_{1}}}\|x-y\|\right)+\max _{x \in \tilde{\gamma}_{s_{1}}}\left(\min _{y \in \gamma_{1}}\|x-y\|\right)
$$

two strainline segments $\gamma_{1}$ and $\tilde{\gamma}_{s_{1}}$ are smaller than a small threshold value $d_{0}$, then discard either $\gamma_{1}$ or $\tilde{\gamma}_{s_{1}}$.

H5 Vary the parameter $s_{1}$ in the definition of the plane family $\Pi\left(s_{1}\right)$ to obtain uniform coverage of the domain of interest. Repeat H1-H4 for each $s_{1}$. Obtain repelling hyperbolic LCSs by fitting a surface to the parametrized curve family $\gamma_{s_{1}}\left(s_{2}\right)$, as described in Remark 4 .

H6 Replace the eigenvector $\xi_{3}$ with $\xi_{1}$ in steps H1-H4 to obtain reduced stretchlines as trajectories of (16). Construct attracting hyperbolic LCSs following step H5.

\subsection{Algorithm for elliptic LCSs}

SH1 Compute the Cauchy-Green strain tensor $C_{t_{0}}^{t_{0}+T}$ and its eigenvectors $\xi_{1}$ and $\xi_{3}$ on a twodimensional grid $\mathcal{G}_{0}$ defined on the reference plane $\Pi\left(s_{1}\right)$

SH3 Pick a sparser grid $\mathcal{G}_{1}$ of initial conditions. Launch reduced shearlines $\gamma_{s_{1}}\left(s_{2}\right)$ as trajectories of the ODE 18, from points of $\mathcal{G}_{1}$ satisfying $\left|H_{n_{ \pm}}\right|=\left|\left\langle\nabla \times \xi_{3}, \xi_{3}\right\rangle\right|<\epsilon_{0}$ for some threshold parameter $\epsilon_{0}$.

SH3 Integrate such reduced shearlines as long as the running average of $\left|H_{n_{ \pm}}\right|$stays below $\epsilon_{0}$.

SH4 Keep only reduced shearlines that form limit cycles.

SH5 Vary the parameter $s_{1}$ in the definition of the plane family $\Pi\left(s_{1}\right)$ to obtain uniform coverage of the three-dimensional domain of interest. Repeat SH1-SH4 for each $s_{1}$. Starting from a closed shearline $\gamma_{s_{1 \min }}\left(s_{2}\right)$ on the plane $\Pi\left(s_{1 \text { min }}\right)$ corresponding to the lowest value of the parameter $s_{1}$, obtain a discretized approximation $\gamma_{s_{1}}\left(s_{2}\right)$ to a closed shear barrier by always selecting the closest closed reduced shearline in the planes $\Pi\left(s_{1}\right)$ under increasing $s_{1}$. Obtain elliptic LCS by fitting a surface to the curve-family $\gamma_{s_{1}}\left(s_{2}\right)$, as described in Remark 4 .

\section{Examples}

\subsection{Steady $A B C$ flow}

As a first example, we consider the steady $\mathrm{ABC}$ flow

$$
\begin{aligned}
& \dot{x}=A \sin z+C \cos y, \\
& \dot{y}=B \sin x+A \cos z, \\
& \dot{z}=C \sin y+B \cos x,
\end{aligned}
$$

an exact solution of Euler's equation. We select the parameter values $A=\sqrt{3}, B=\sqrt{2}$ and $C=1.0$. This well-studied set of parameter values yields the Poincare map shown in Fig. 2. We first use the theory developed here to construct the full two-dimensional transport barriers suggested by this Poincare map. Because the flow is steady, the transport barriers we seek are also invariant manifolds in the phase space, not just in the extended phase space.

We therefore only need to carry out the computational steps H1-H4 and SH1-SH4 of Section 4 to obtain intersection curves between barriers and a single reference plane $\Pi$. We then advect these intersection curves under the flow map to obtain the full two-dimensional barriers.

In Sections 5.2 and 5.3, we consider temporally periodic and aperiodic versions of (20) where this simple approach will no longer suffice. The present steady case is only considered here to provide a consistency check on a well-studied steady flow. 


\subsubsection{Elliptic LCSs in the steady ABC flow}

We first perform the computational steps SH1-SH4 of the previous section in one of the vortical regions seen in the Poincare map plot of Fig. 2. The lower panels of the same figure show orbits the Poincare map in blue (color only in the online version), as well as closed reduced shearlines (green) obtained from the computational steps SH1-SH4 on the plane

$$
\Pi=\{(x, y, z): z=0\}
$$

or two different integration times. In both cases, a uniform grid $\mathcal{G}_{0}$ of $1000 \times 1000$ initial points was used to compute the Cauchy-Green strain tensor. The tolerance parameter in the computational step SH3 is chosen to be $\epsilon_{0}=10^{-2}$.

While the plots in Fig. 2 show curves in the $(x, y)$ plane, the analysis is inherently threedimensional. Indeed, computing the vector $n_{ \pm}$in equations (18)-(19) requires fully three-dimensional trajectory integration.
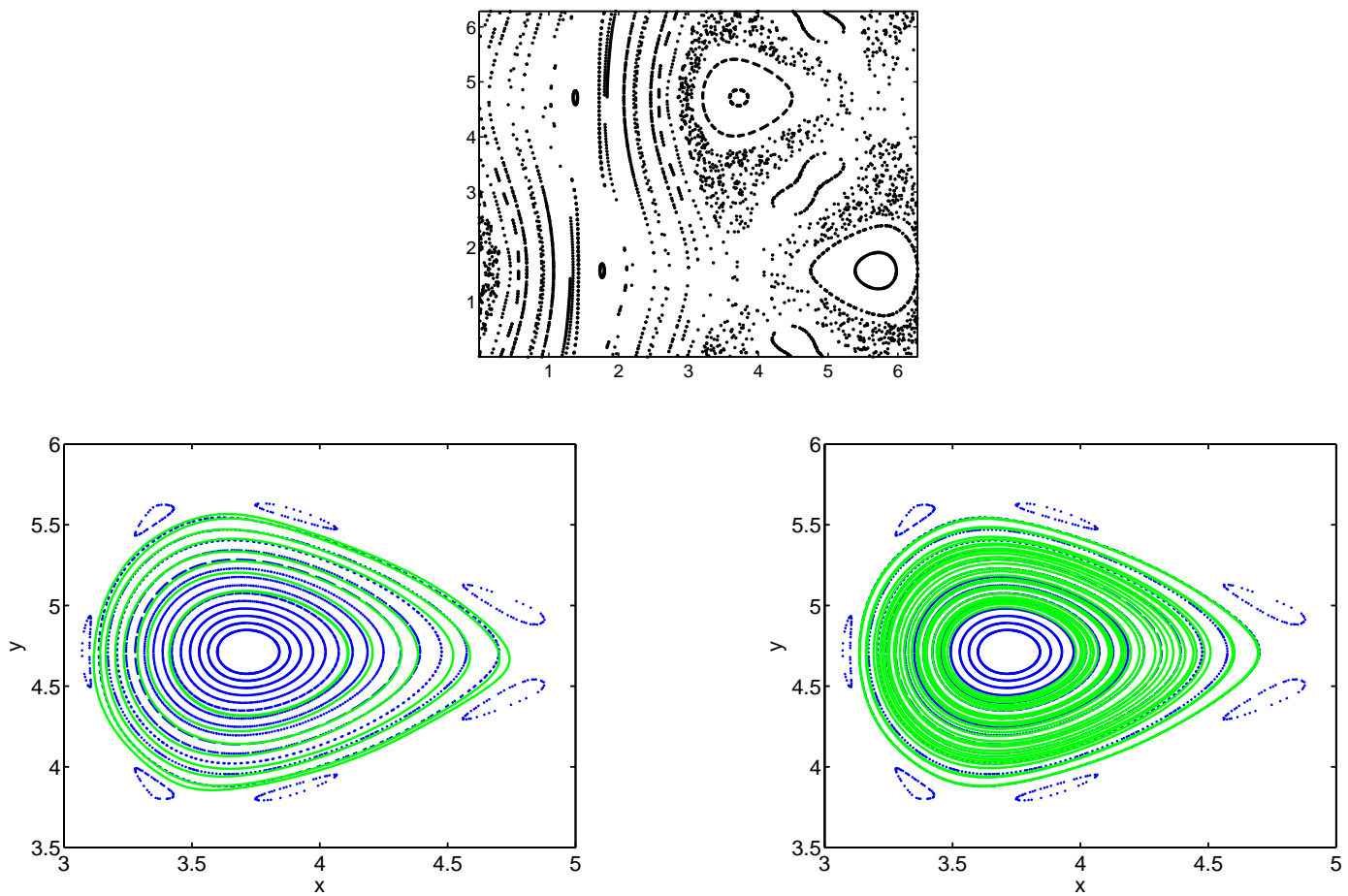

Figure 2: Top: Poincare map for the steady ABC flow on the $z=0$ plane. Bottom: Closed reduced shearlines on the plane $z=0$ that approximate invariant tori for the steady $\mathrm{ABC}$ flow. The time interval used in their construction was $[0,40]$ for the left panel and $[0,150]$ for the right panel.

By the periodic nature of the phase space, the shear LCS obtained from the advection of closed, reduced shearlines are two-dimensional tori. To bring out the toroidal nature of these barriers, we introduce new coordinates with the help of the approximate spatial core $\left(x_{0}(z), y_{0}(z), z\right)$ obtained by advecting the vortical center point of Fig. 2. Using this center curve, we introduce the toroidal coordinate system

$$
\begin{aligned}
& \bar{x}=\left[x-x_{0}(z)+R_{1}\right] \cos (z), \\
& \bar{y}=\left[x-x_{0}(z)+R_{1}\right] \sin (z), \\
& \bar{z}=R_{2}\left[y-y_{0}(z)\right],
\end{aligned}
$$

where $R_{i}$ are positive constants. A nested family of invariant tori obtained from this transformation is shown in Fig. 3. 


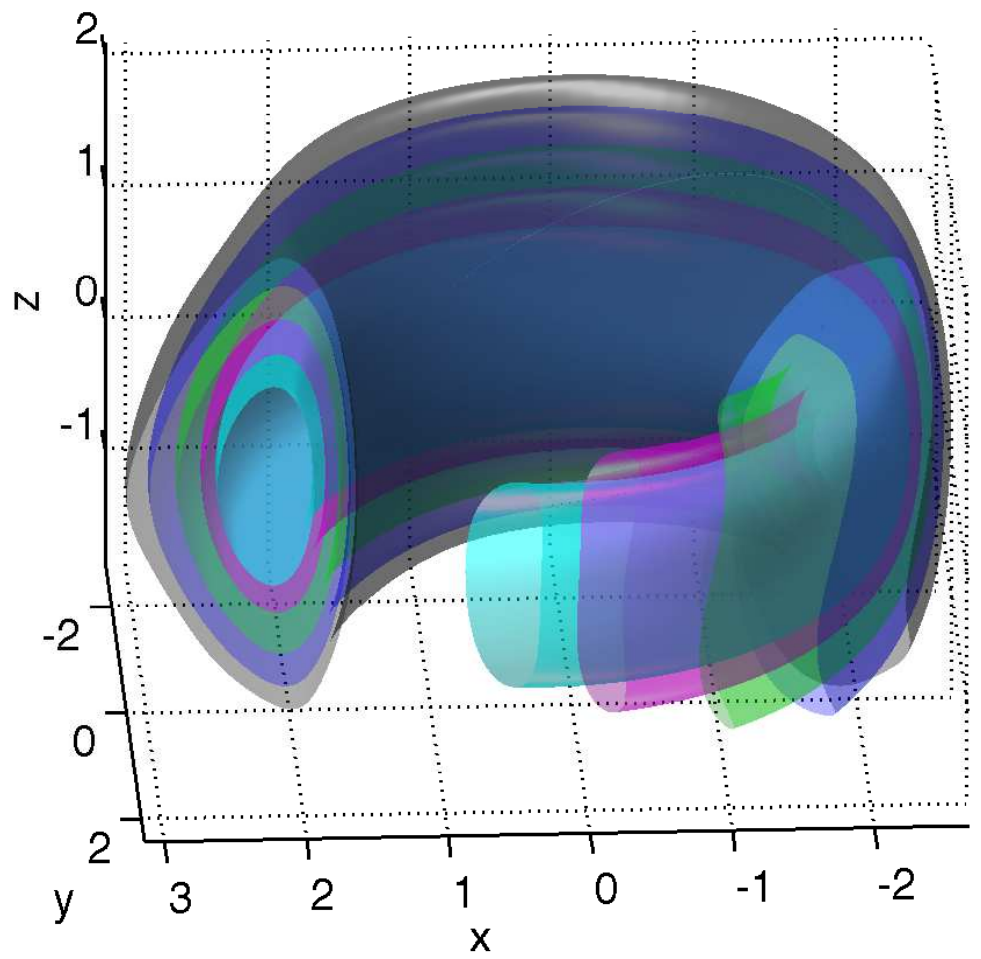

Figure 3: An embedding of a nested elliptic LCS family in the steady ABC flow. These LCSs approximate invariant tori from a finite-time observation over the time interval $[0,40]$.

Fig. 4 shows the shear helicities $H_{n_{ \pm}}$along the line segment $y=4.7$, with dots marking the locations of closed shearlines obtained from our finite-time analysis. Despite the significant numerical noise in the computation of the shear helicity, the zeros of $H_{n_{ \pm}}$move closer and closer to the computed shearlines, validating these shearlines as curves on a shear LCS in the sense of Definition 1.

\subsubsection{Repelling hyperbolic LCSs in the steady ABC flow}

We compute the Cauchy-Green strain tensor $C_{0}^{3}$ over a $500 \times 500$ grid on the plane $z=0$. The tolerance parameter in the computational step H3 is chosen to be $\epsilon_{0}=10^{-4}$. Under this tolerance level, intersections or repelling hyperbolic LCSs with the $z=0$ plane are shown in the left panel of Fig. 5, obtained as parametrized curves. Also shown is the vertical line $y=0.5$, along which we compute the strain helicity $H_{\xi_{3}}$ (cf. the right panel of Fig. 5). The latter figure illustrates that the reduced strainlines shown in the left panel are indeed intersections of the $z=0$ plane with repelling hyperbolic LCSs in the sense of Definition 1.

To illustrate the dynamical impact of the barrier surface emanating from the reduced strainlines, we select one of these strainlines (shown in green in the left panel of Fig. 6). We perturb this reduced strainline segment in the $x$ direction by \pm 0.01 to obtain the blue and red curves shown in the same panel. We then advect all three parameterized curves from $t_{0}=0$ to $t_{0}+T=3$ to obtain the surfaces shown in the left panel of Fig. 6 . Note that the blue and red curves have noticeable upward and downward $z$-drifts, respectively, while the surface evolving from the green reduced strainline has no $z$-drift.

\subsection{Time-periodic $A B C$ flow}

We now consider a temporally periodic version of the $\mathrm{ABC}$ flow, given by 

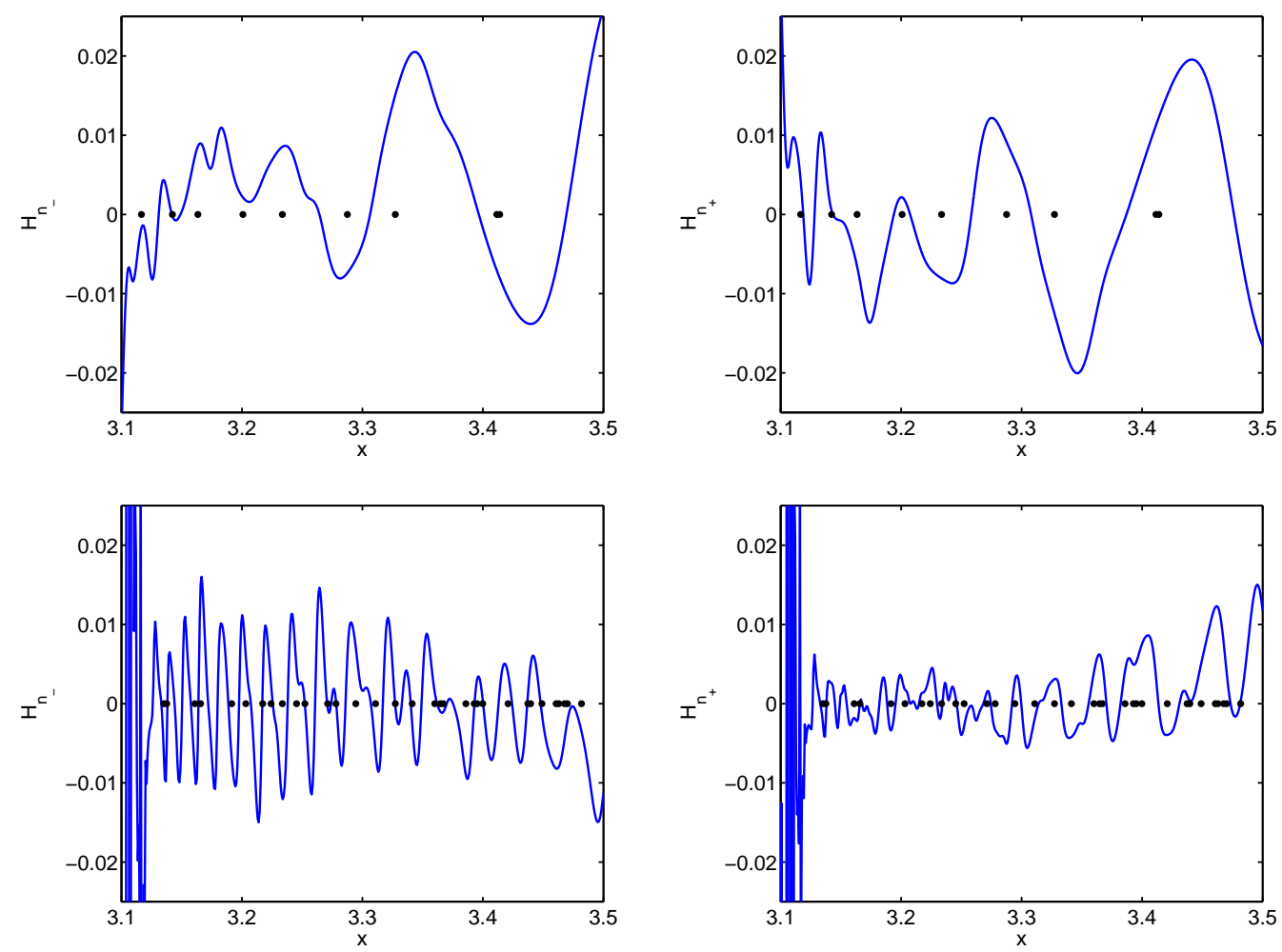

Figure 4: Upper panels: The helicities $H_{n_{ \pm}}$of the shear vector fields $n_{ \pm}$, respectively, along the line $y=4.7$ for integration length $T=40$. Black dots indicate the $x$ coordinate of the closed shearlines shown in Fig. 2. Lower panels: the same for integration time $T=150$.

$$
\begin{aligned}
& \dot{x}=(A+0.1 \sin t) \sin z+C \cos y, \\
& \dot{y}=B \sin x+(A+0.1 \sin t) \cos z, \\
& \dot{z}=C \sin y+B \cos x .
\end{aligned}
$$

The first return map to the plane $z=0$ is now a non-autonomous map. Therefore, to gain insight into the flow from classical tools, only a fully three-dimensional temporal Poincare map can be used. This would result in spatially scattered points, as opposed to the sharply defined shear and hyperbolic barriers that we will continue to obtain form our approach.

\subsubsection{Elliptic LCSs in the time-periodic ABC flow}

We compute the Cauchy-Green strain tensor $C_{0}^{30 \pi}$ over a $500 \times 500$ grid in the plane $z=0$. This integration length is equal to 15 iterations of the temporal Poincare map $F_{0}^{2 \pi}$. The tolerance parameter in the computational step SH3 is chosen to be $\epsilon_{0}=10^{-2}$. Fig. 7 shows the closed reduced shearlines we find as limit cycles of the equation (18). The shear-helicity zero distribution along these curves is similar to that in the steady case, and hence is omitted here for brevity.

Next, we iterate the outermost closed reduced shearline under the Poincare map $F_{0}^{2 \pi}$. The result is a two-dimensional invariant torus for $F_{0}^{2 \pi}$, shown in Fig. 7 under the embedding (21). This torus is an intersection of a three-dimensional invariant torus of the full, spatially and temporally periodic flow (defined over the toroidal phase space $\mathbb{T}^{4}$ ) with the $t=0$ hyperplane.

We now illustrate the barrier property of the three-dimensional torus represented by the elliptic LCS of Fig. 7. To this end, we advect two initial conditions from the interior of the two-dimensional 

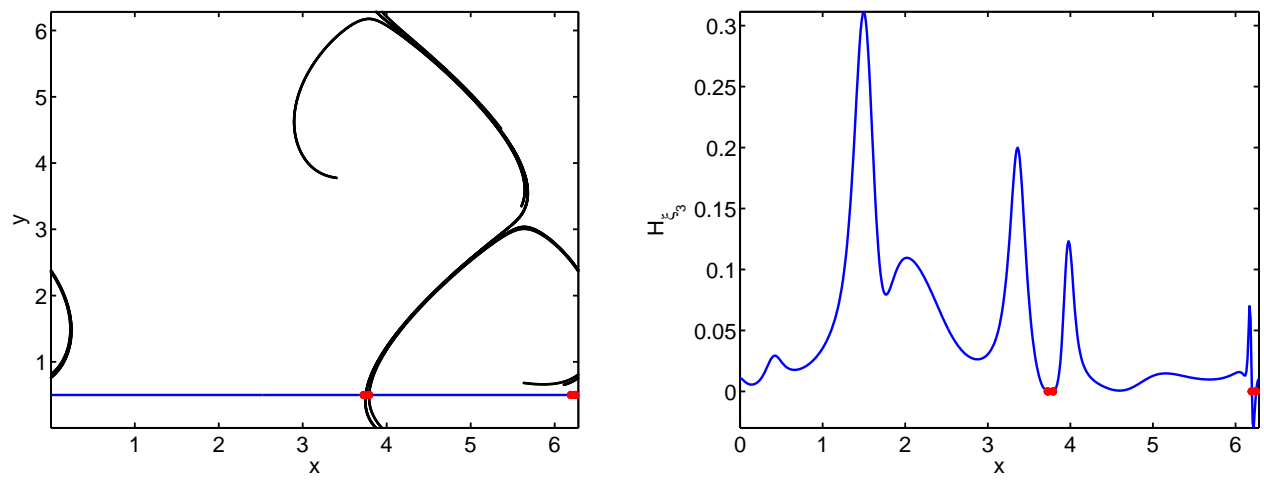

Figure 5: Left panel: Reduced strainlines of minimal average helicity, with the line $y=0.5$ shown in blue, and with red dots indicating points where the helicity $H_{\xi_{3}}$ is exactly zero. Right panel: the helicity $H_{\xi_{3}}$ plotted as a function of $x$ along the line $y=0.5$, with its zeros highlighted in red.
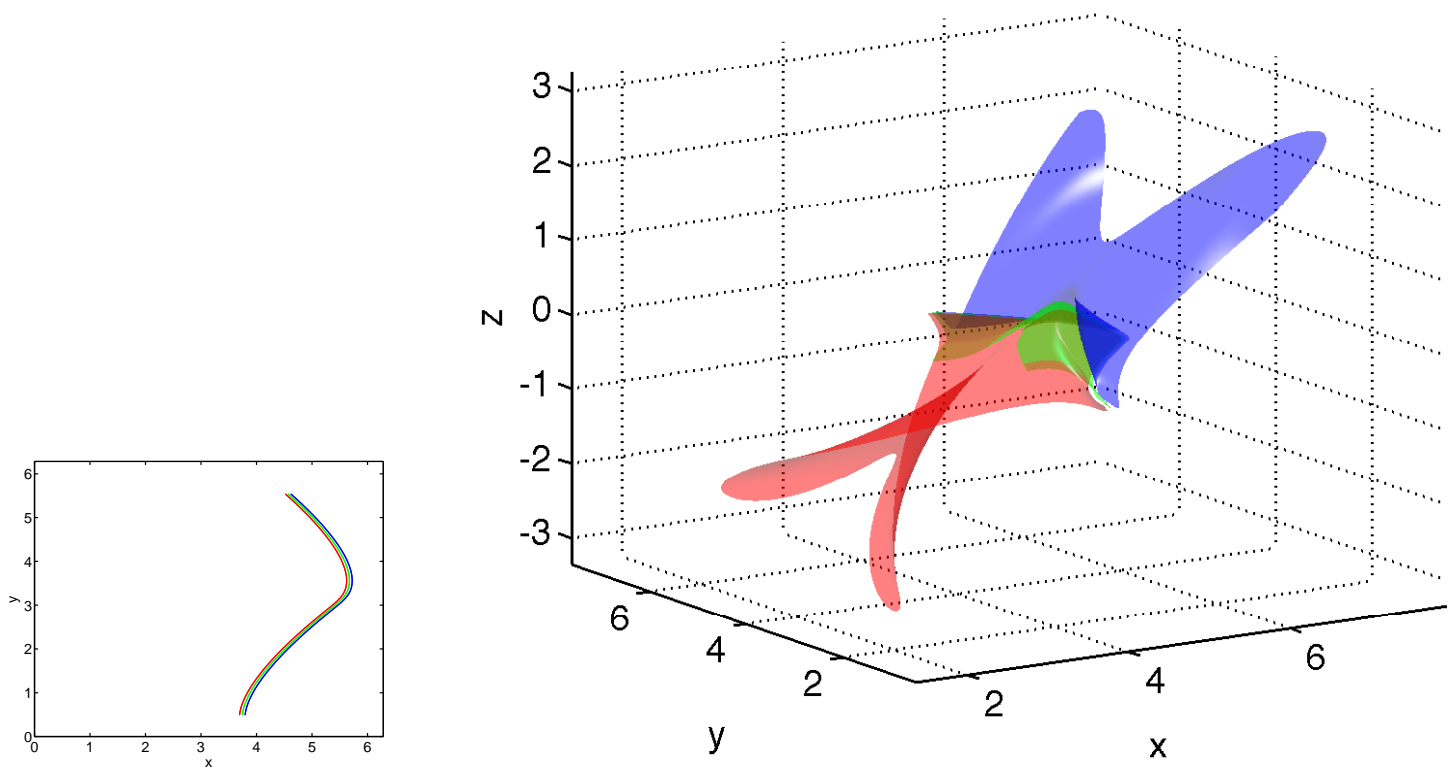

Figure 6: Left panel: reduced strainline of zero helicity (green), and its perturbation to the left (red) and to the right (blue) by 0.01. Right panel: Invariant surfaces through these three curves obtained by advection under the flow map $F_{0}^{30}$. They illustrate the repelling barrier property of the green LCS through the green reduced strainline. 

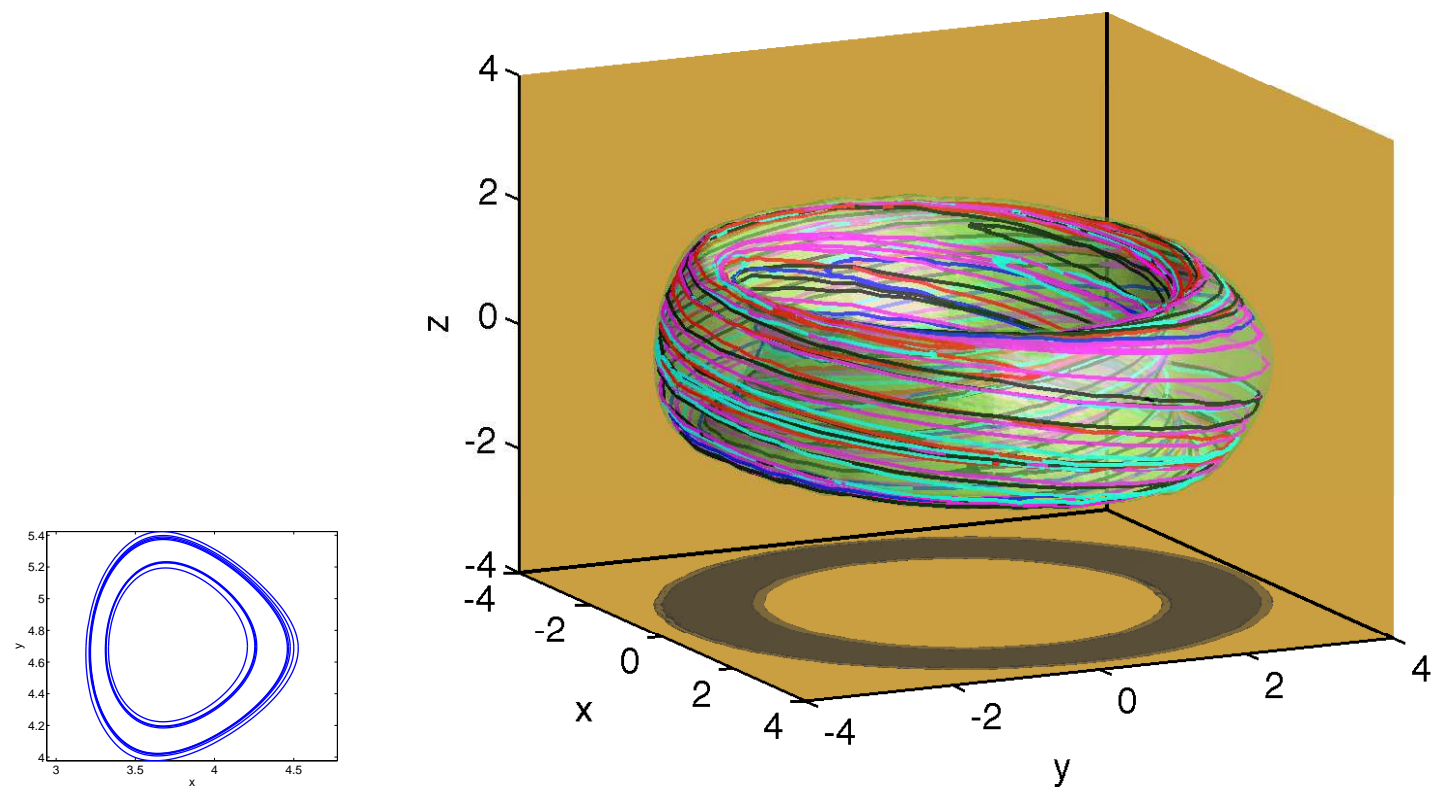

Figure 7: Left: Closed reduced shearlines for the time-periodic ABC flow on the plane $z=0$, constructed from flow data over the time interval $\left[t_{0}, t_{0}+T\right]=[0,30 \pi]$. Right panel: invariant torus as elliptic LCS for the temporal Poincare map, obtained from subsequent images of the outermost closed shearline under iterations of $F_{0}^{2 \pi}$. (The blue, red, cyan, black, and magenta curves represent the $n$th iterate of $F_{0}^{2 \pi}$ for $n=50,75,110,160,200$, respectively). To illustrate the invariance of the underlying torus, we also computed 400 iterates of the outermost closed shearline under $F_{0}^{2 \pi}$, obtaining the green, filamentation-free toroidal surface.

torus starting from the $z=0$ reference plane, and two other initial conditions from the exterior of this torus within the same plane. These four initial conditions are placed on the grey circle shown in the left panel of Fig. 7. The center of this circle is on the outermost torus barrier, and is advected as a blue trajectory. As seen in the right panel of Fig. 7, the blue trajectory indeed remains confined to a quasi-periodically deforming transport barrier in phase space. This barrier keeps both the red and the yellow initial conditions from spreading. In contrast, the green and black initial conditions launched from outside the outermost torus barrier develop large excursions over time.

\subsubsection{Repelling hyperbolic LCSs in the time-periodic ABC flow}

We now compute repelling hyperbolic barriers for the time-periodic ABC flow using the slicing method described in steps H1-H5 of section 4.1. We select the discrete family of 21 planes

$$
\Pi\left(s_{1}\right)=\left\{(x, y, z) \in[0,2 \pi]^{3}: z=s_{1}\right\}, \quad s_{1}=0.00,0.005,0.01, \ldots, 0.1,
$$

with a $500 \times 500$ uniform grid $\mathcal{G}_{0}$ within each such plane. Over the time interval ranging from $t_{0}=0$ to $t_{0}+T=4.0$, we carried out the procedure outlined in steps H1-H5 of section 4 . In step H2, the grid $\mathcal{G}_{1}$ was chosen as $600 \times 10$ in $(x, y)$, and the helicity parameter was chosen as $\epsilon_{0}=10^{-4}$. The filtered reduced strainlines obtained from $\mathrm{H} 1-\mathrm{H} 4$ on the $\Pi(0)$ plane are shown in the left panel of Fig. 9. The right panel of the same figure shows the reconstructed barrier surface by performing step $\mathrm{H} 5$ across the plane family $\Pi\left(s_{1}\right)$ and interpolating smooth surfaces over the resulting reduced strainline segments.

\subsection{Chaotically forced $A B C$ flow}

Here we consider a temporally aperiodic version of the $\mathrm{ABC}$ flow, given by the equations 

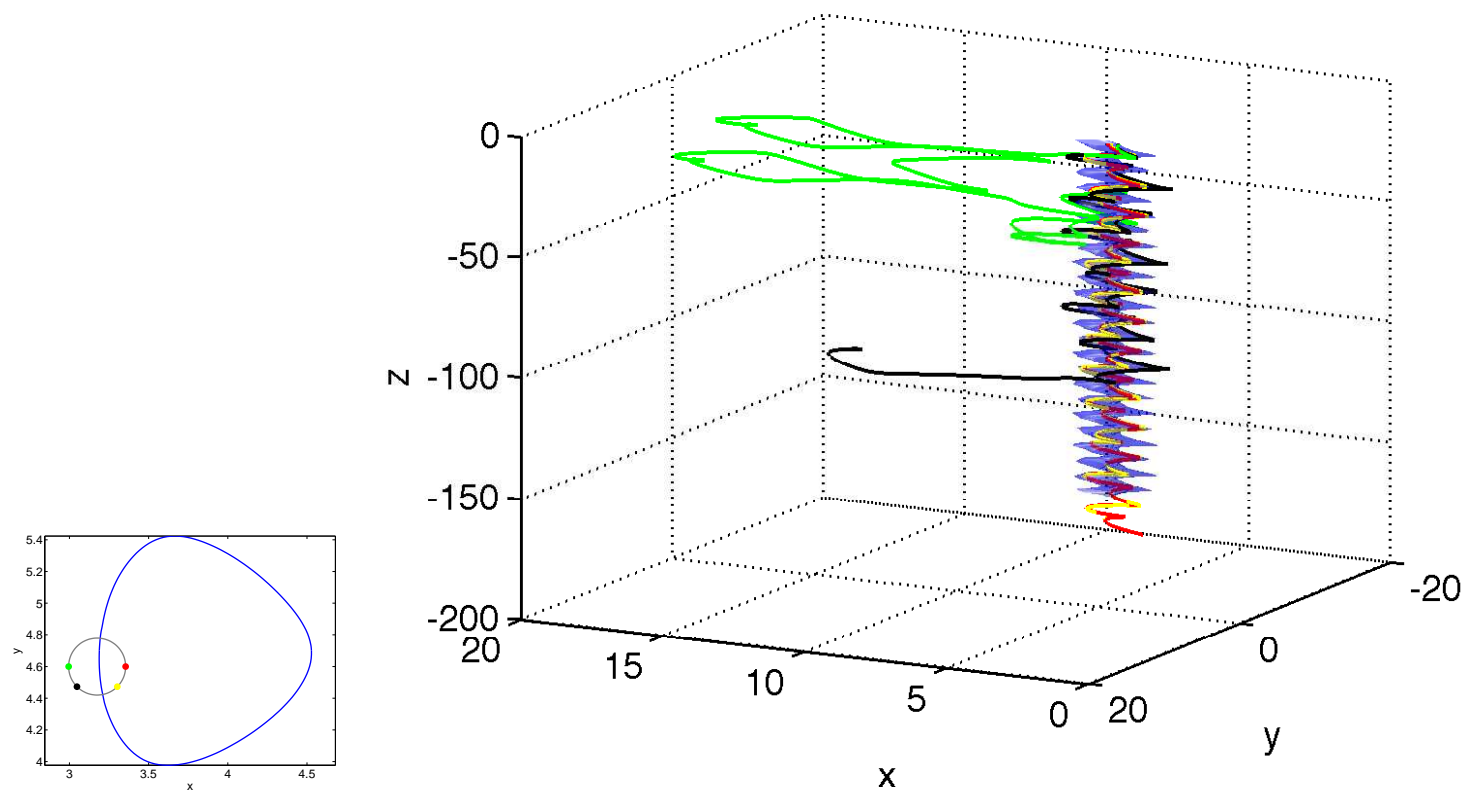

Figure 8: Verification of the quasiperiodic transport barrier obtained from reduced shearlines. Tracers launched inside (red and yellow), along (blue), and outside (green and black) the outermost closed reduced shearline show markedly different behavior in phase space (blue). The time interval of advection was $[0,30 \pi]$.

$$
\begin{aligned}
& \dot{x}=(A+F(t)) \sin z+C \cos y, \\
& \dot{y}=B \sin x+A(A+F(t)) \cos z, \\
& \dot{z}=C \sin y+B \cos x,
\end{aligned}
$$

with $F(t)$ representing a chaotic signal. The signal is generated by a trajectory close to the strange attractor of a periodically forced and damped Duffing oscillator (see Fig. 10). The temporally aperiodic flow (23) admits neither a well-defined spatial nor a well-defined temporal autonomous first return map. Therefore, the simplified barrier visualization methods used for elliptic barriers in the steady (Fig. 3) and time-periodic (Fig. 7) ABC flows are no longer applicable.

\subsubsection{Elliptic LCSs in the chaotically forced $A B C$ flow}

We compute the Cauchy-Green strain tensor $C_{0}^{100}$ over a $500 \times 500$ grid in each member of the plane family

$$
\Pi\left(s_{1}\right)=\left\{(x, y, z) \in[0,2 \pi]^{3}: z=s_{1}\right\}, \quad s_{1}=2 k \pi / 150, \quad k=0,1,2, \ldots, 149 .
$$

The forthcoming computations were carried out in a parallelized fashion over the $150 s_{1}$-slices defined in The closed reduced strainlines obtained form SH1-SH4 on the $\Pi(0)$ plane are shown in the upper left panel of Fig. 11. The tolerance parameter in the computational step SH3 is chosen to be $\epsilon_{0}=10^{-2}$. The upper right panel of the same figure shows the reconstructed outermost elliptic LCS by performing step SH5 across the plane family $\Pi\left(s_{1}\right)$ and interpolating smooth surfaces over the resulting closed shearline segments. The lower left panel of the figure confirms the coherence of the detected barrier up to time 100. The lower right panel of the figure shows that the extracted barrier remains coherent under advection even at time 150.

The time interval used in verifying sustained coherence for the elliptic LCS in the lower right panel of Fig. 11 is 50\% longer than the time interval used to extract this barrier. This sustained 


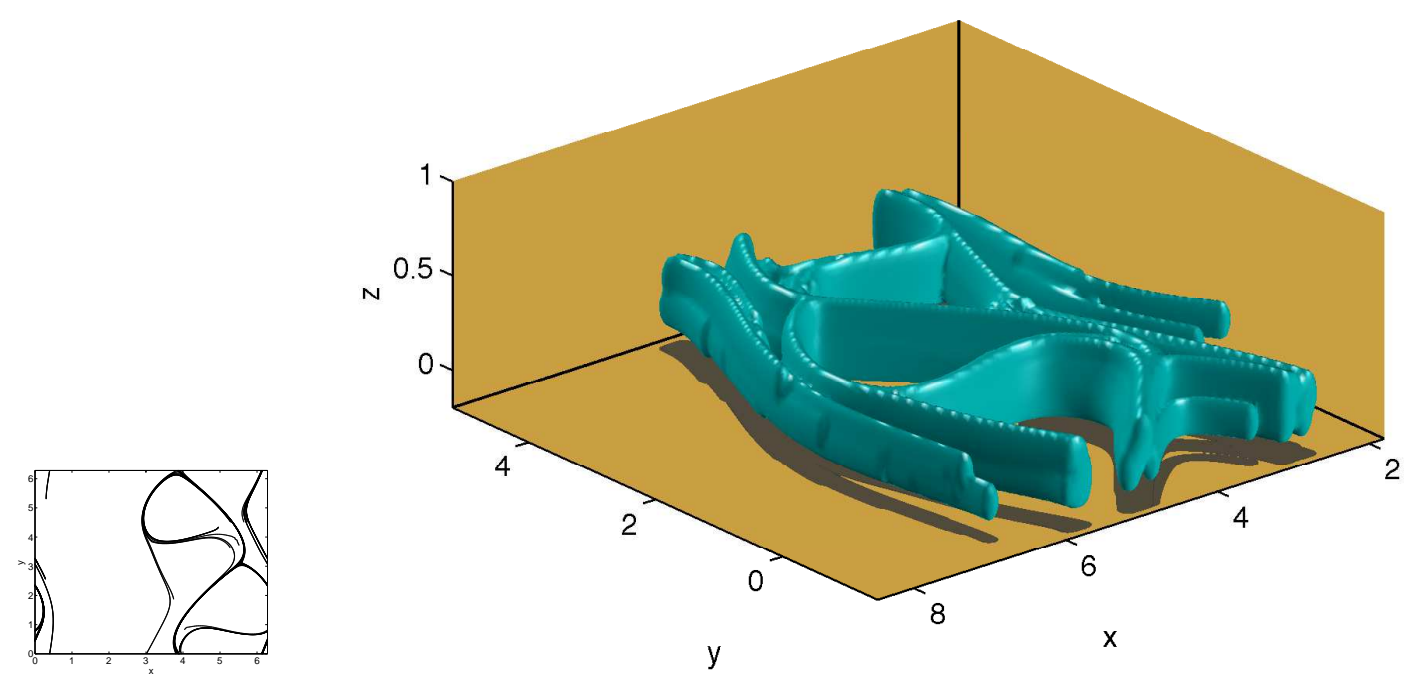

Figure 9: Left: Reduced strainlines on the $\Pi(0)$ plane for the temporally periodic $A B C$ flow for the time interval $[0.0,4.0]$. Right: repelling hyperbolic LCSs obtained by computing reduced strainlines over the plane family $\Pi\left(s_{1}\right)$.

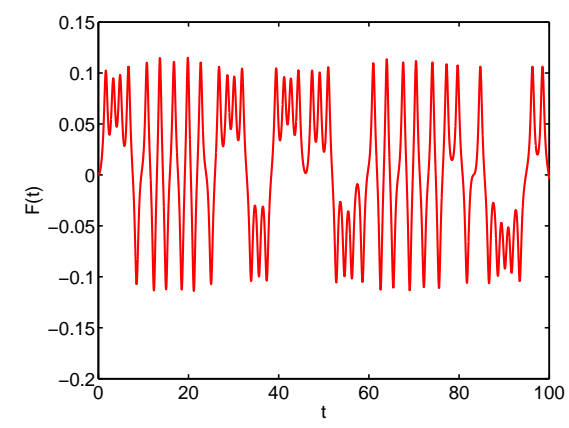

Figure 10: Aperiodic forcing used in the temporally aperiodic ABC-type flow 23.

coherence property is remarkable, as illustrated by Fig. 12. In this figure, a circle of one million initial conditions is selected as a perturbation to the smaller diameter of the torus barrier. Just after an advection time of $t=13.0$, the ring quickly loses all its coherence, stretching and folding by a large amount in a visibly chaotic fashion.

Fig. 13 shows the same type of verification of the optimality of the barrier that we employed in Fig. 8 for the time-periodic ABC flow. Again, tracers launched inside the barrier remain confined to the interior of the barrier, while tracers launched slightly outside the barrier exhibit large excursions.

\subsubsection{Repelling hyperbolic barriers in the chaotically forced $A B C$ flow}

Finally, we compute repelling hyperbolic barriers for the chaotically forced $\mathrm{ABC}$ flow using steps H1-H5 of section 4. The Cauchy-Green strain tensor $C_{0}^{5}$ is computed over the same plane family used in section 5.2.2 for the time-periodic case. The grids $\mathcal{G}_{0}$ and $\mathcal{G}_{1}$, as well as the admissible upper bound $\epsilon_{0}$ on the helicity norm, are also selected the same as in section 5.2.2. Fig. 14 shows the final result, the set of extracted repelling hyperbolic barriers in the chaotically forced case. 

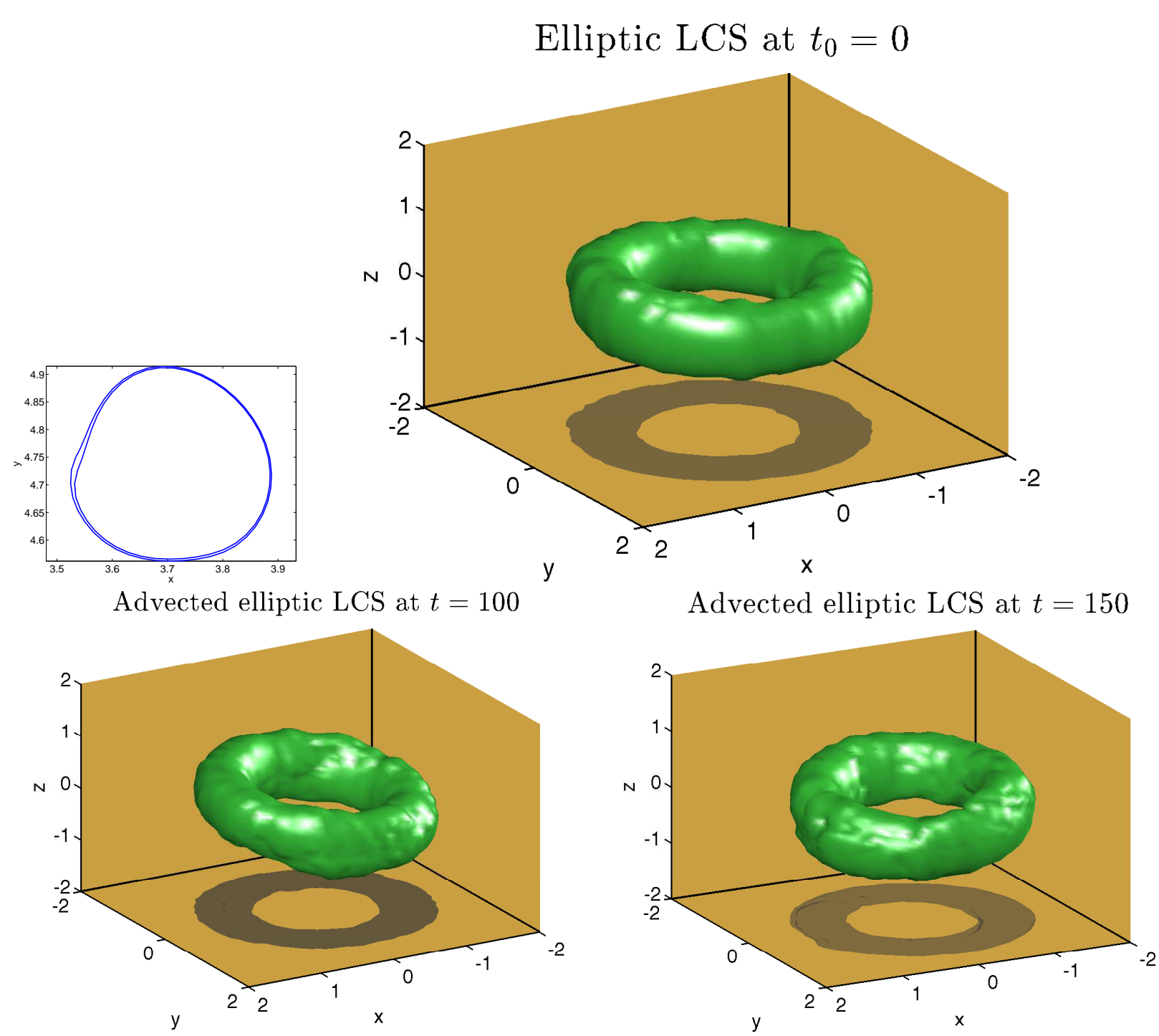

Figure 11: Elliptic LCS in the chaotically forced ABC flow. Upper left: Reduced closed shearlines in the $\Pi(0)$ (i.e., $z=0$ ) plane computed from $C_{0}^{100}$. Upper right: Outermost elliptic LCS at time $t_{0}=0$, visualized through the torus embedding (21). Lower left: Advected elliptic LCS at time $t_{0}+T=100$, the final time used in its construction. Lower right: Advected elliptic LCS at time $t_{0}+T=150$ which is larger $50 \%$ larger than the final time used in its construction.

\section{Conclusions}

We have developed a unified theory of transport barriers for three-dimensional unsteady flows. The barriers are attracting, repelling or shear LCS, which are constructed to prevail as skeletons of material deformation over a given finite time interval of observation. Out of general shear LCSs, elliptic LCSs are distinguished by their tubular or toroidal topology. Our approach renders all these LCSs as explicitly parametrized surfaces with precisely understood impact on neighboring material blobs. This is to be contrasted with alternative approaches that seek the domains separated by transport barriers from various indicator functions, without yielding specific dynamical information or a parametrization for the barrier itself.

Our approach closely reproduces known transport barriers in the steady ABC flow, and provides similar results for time-periodic and time-aperiodic version of the same flow. Remarkably, generalized 

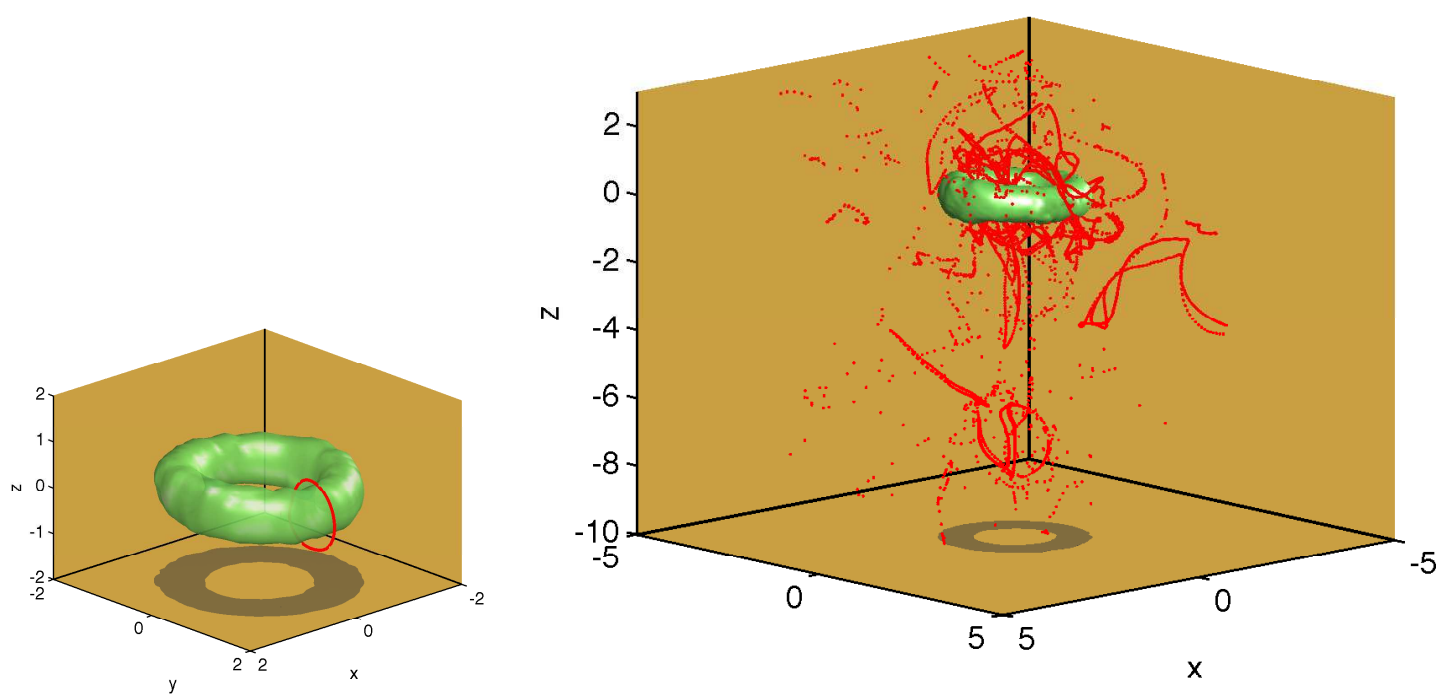

Figure 12: Evolution of an elliptic LCS and of a ring placed near the LCS at time $t_{0}=0.0$ (left) into their final position at time $t=13.0$ (right).
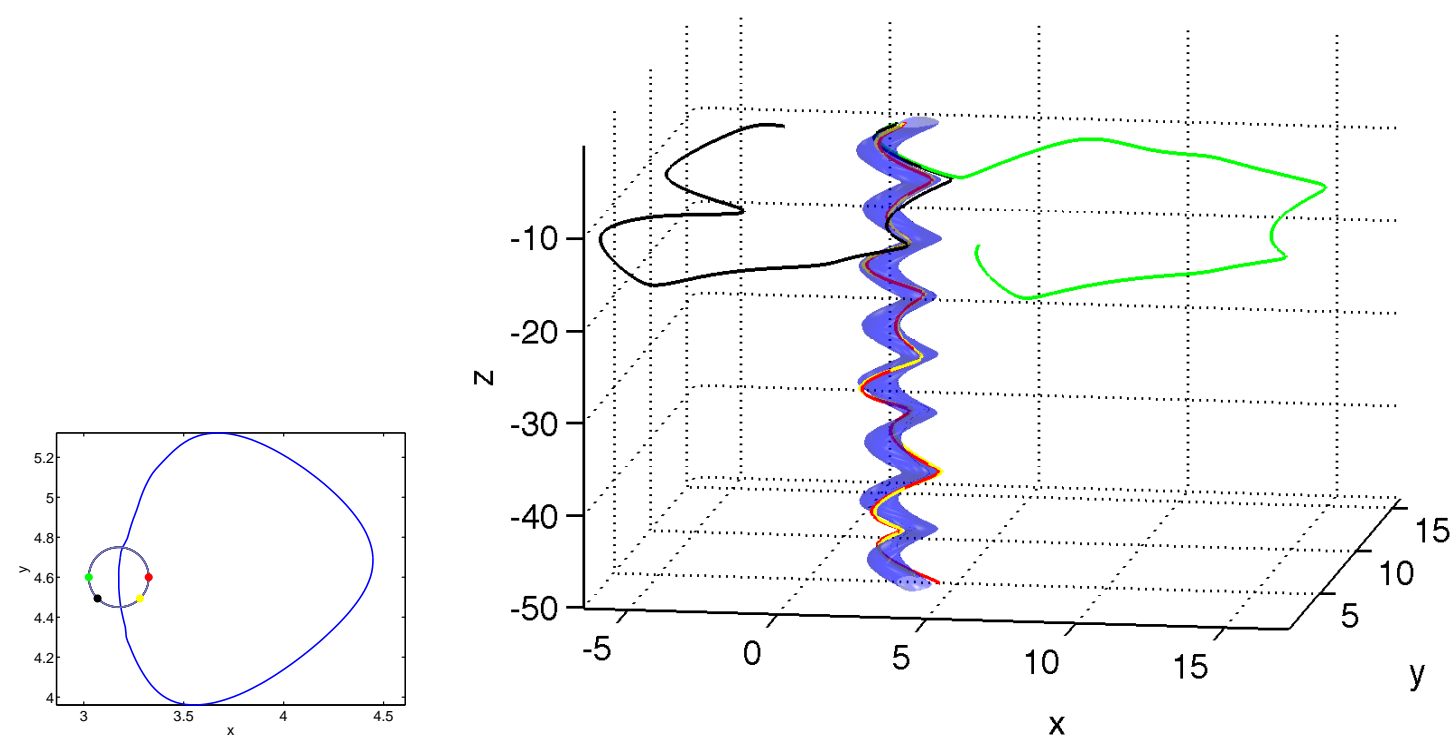

Figure 13: Advection of tracers inside (red and yellow) and outside (green and black) of the outermost closed shearline (blue) under the flow map $F_{0}^{25}$. 

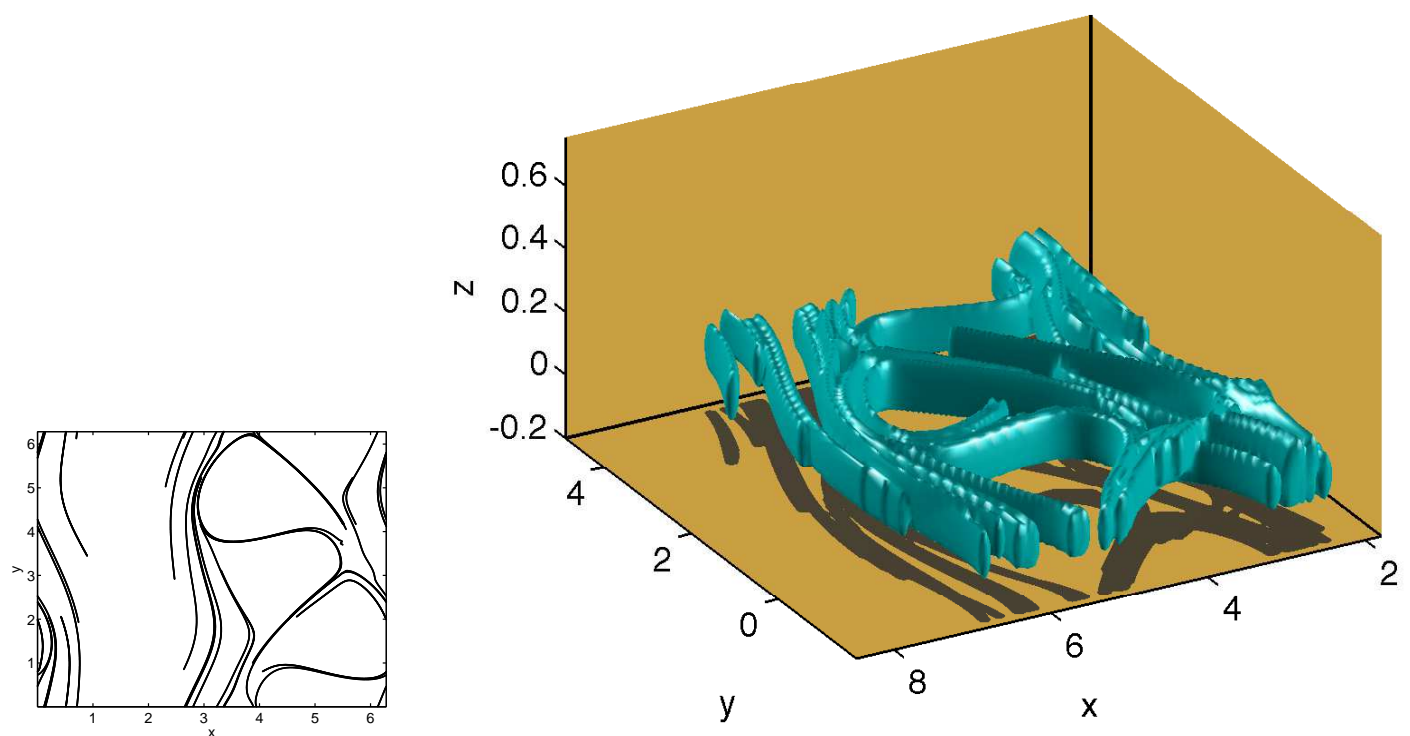

Figure 14: Reduced strainlines on the $z=0$ planes (left) and repelling hyperbolic LCSs at time $t_{0}=0$ (right) for the chaotically forced ABC flow, reconstructed from the flow map $F_{0}^{5}$.

KAM tori (Lagrangian vortex rings) continue to exist in the general aperiodic case, providing sharp boundaries for coherent toroidal islands in an otherwise chaotic flow.

Although similar notions of multi-dimensional hyperbolic LCSs have been used earlier [15], the theory of elliptic LCS as well as the computational methodology developed here for all types of LCSs is new. Our notion of an ellkiptic LCS extends that arising in the two-dimensional theory of shear barriers developed in [16]. This two-dimensional theory has identified highly coherent Lagrangian eddies from satellite-mapped surface velocities in the Agulhas leakage of the Southern Ocean [6]. The direct analogy with the two-dimensional theory promises similar results in the application of the present techniques to three-dimensional numerical or experimental flow data.

\section{Appendix A. Shear LCSs in unsteady, three-dimensional parallel shear flows}

Consider the flow

$$
\begin{aligned}
& \dot{x}(t)=u(z, t), \\
& \dot{y}(t)=v(z, t), \\
& \dot{z}(t)=w(t),
\end{aligned}
$$

where the dependence of $u, v$, and $w$ on their arguments is smooth but otherwise arbitrary. Trajectories of (A.1) satisfy

$$
\begin{aligned}
& x(t)=x_{0}+\int_{t_{0}}^{t_{0}+T} u(z(\tau), \tau) d \tau, \\
& y(t)=y_{0}+\int_{t_{0}}^{t_{0}+T} v(z(\tau), \tau) d \tau, \\
& z(t)=z_{0}+\int_{t_{0}}^{t_{0}+T} w(\tau) d \tau .
\end{aligned}
$$


We introduce the functions $a\left(z_{0}, t_{0}, T\right)$ and $b\left(z_{0}, t_{0}, T\right)$ as

$$
\begin{aligned}
& a\left(z_{0}, t_{0}, T\right)=\int_{t_{0}}^{t_{0}+T} u_{z}(z(\tau), \tau) d \tau, \\
& b\left(z_{0}, t_{0}, T\right)=\int_{t_{0}}^{t_{0}+T} u_{z}(z(\tau), \tau) d \tau .
\end{aligned}
$$

Suppressing the arguments of $a$ and $b$, we obtain the Cauchy-Green strain tensor in the form

$$
C_{t_{0}}^{t_{0}+T}=\left(\begin{array}{ccc}
1 & 0 & a \\
0 & 1 & b \\
a & b & a^{2}+b^{2}+1
\end{array}\right) .
$$

We now show that the planes $z=k=$ const. are shear LCSs in the sense of Definition 1 , as obtained from an application of Theorem 1. To do this, we use an expression for the angle $\phi$ that the vector $v_{ \pm}:=\xi_{2} \times n_{ \pm}$encloses with the vertical planes $z=k$ for general 3D flows.

Lemma 1. Consider a general three-dimensional unsteady flow, and letM

$$
v_{ \pm}=\xi_{2} \times n_{ \pm}=(\sin \phi \cos \theta, \sin \phi \sin \theta, \cos \phi) .
$$

Also, let $C_{i j}$ denote the $(i, j)$-th entry of the Cauchy-Green strain tensor $C_{t_{0}}^{t_{0}+T}$. We then have

$$
\begin{aligned}
& C_{11} \sin ^{2} \phi \cos ^{2} \theta+C_{22} \sin ^{2} \phi \sin ^{2} \theta+C_{33} \cos ^{2} \phi \\
& +2\left(C_{12} \sin ^{2} \phi \sin \theta \cos \theta+C_{13} \sin \phi \cos \phi \cos \theta+C_{23} \sin \phi \cos \phi \sin \theta\right)=\sqrt{\lambda_{1} \lambda_{3}} .
\end{aligned}
$$

Proof. The two sides of equation (A.5) represent two different ways of computing $\left\langle v_{ \pm}, C_{t_{0}}^{t_{0}+T} v_{ \pm}\right\rangle$. The left-hand side is computed using the matrix elements of $C_{t_{0}}^{t_{0}+T}$. To compute the same quantity and arrive at the quantity on the right-hand side, recall first that $n_{ \pm}=\alpha \xi_{1} \pm \beta \xi_{3}$, where

$$
\alpha=\sqrt{\frac{\sqrt{\lambda_{1}}}{\sqrt{\lambda_{1}}+\sqrt{\lambda_{3}}}}, \quad \beta=\sqrt{\frac{\sqrt{\lambda_{3}}}{\sqrt{\lambda_{1}}+\sqrt{\lambda_{3}}}} .
$$

Hence $v_{ \pm}=\alpha \xi_{3} \pm \beta \xi_{1}$, implying

$$
\left\langle v_{ \pm}, C_{t_{0}}^{t_{0}+T} v_{ \pm}\right\rangle=\alpha^{2} \lambda_{3}+\beta^{2} \lambda_{1}=\sqrt{\lambda_{1} \lambda_{3}}
$$

which proves the lemma.

For the unsteady parallel shear flow defined by (A.1), one can verify that $\lambda=1.0$ is an eigenvalue of the Cauchy-Green strain tensor (A.4) with eigenvector $\xi=\left(-\omega_{2}^{\prime}\left(z_{0}\right) / \omega_{1}^{\prime}\left(z_{0}\right), 1,0\right)$. Moreover, symbolic computations in MATLAB show that the other eigenvalues of (A.4) are greater than one, or less than one. More specifically, another eigenvalue of $C_{t_{0}}^{t_{0}+T}$ is $\lambda=\left(\left(a^{2}+b^{2}\right)\left(a^{2}+b^{2}+4\right)\right)(1 / 2) / 2+$ $a^{2} / 2+b^{2} / 2+1$, which shows that as long as $a$ and $b$ are both nonzero, $C_{t_{0}}^{t_{0}+T}$ will have an eigenvalue greater than one. By incompressibility, another eigenvalue is then less than one. Thus $\lambda_{2}=1$, and $\xi_{2}$ is parallel to the plane $z=k$.

Since $\xi_{2}$ is always orthogonal to $n_{ \pm}$, to show that $z=k$ is a shear LCS, it remains to argue that $v_{ \pm}$is also tangent to $z=k$. Since the flow is incompressible, we conclude that $\lambda_{1} \lambda_{3}=1$. As a result, the right-hand side of the angle formula in Lemma 1 is one. Using our specific form of the Cauchy-Green strain tensor (A.4), the angle formula (A.5) becomes

$$
\cos \phi\left[\left(a^{2}+b^{2}\right) \cos \phi+2 \sin \phi(a \cos \theta+b \sin \theta)\right]=1,
$$

which has $\phi=0$ as a solution. Therefore, we conclude that both $\xi_{2}$ and $v_{ \pm}=n_{ \pm} \times \xi_{2}$ are tangent to the plane $z_{0}=k$, which is therefore a shear LCS provided that $a\left(z_{0}, t_{0}, T\right) \neq 0$ or $b\left(z_{0}, t_{0}, T\right) \neq 0$. 


\section{Appendix B. Evolution of LCS surface area}

We consider how the surface area of an LCS changes under the flow map. We have the following general result

Lemma 2. [Surface area of a general material surface] Let $\mathcal{M}(t)$ be a material surface, and $p\left(s_{1}, s_{2}\right)$ be a local parameterization of $\mathcal{M}\left(t_{0}\right)$, where $\left(s_{1}, s_{2}\right)$ lie in a connected open bounded subset $U \subset \mathbb{R}^{2}$. Then the surface area of $F_{t_{0}}^{t_{0}+T}(U)$ can be computed as

$$
S\left(F_{t_{0}}^{t_{0}+T}(U)\right)=\int_{U}\left|\operatorname{det}\left(\nabla F_{t_{0}}^{t_{0}+T}\right)\right| \cdot \sqrt{\left|\left\langle p_{s_{1}} \times p_{s_{2}},\left(C_{t_{0}}^{t_{0}+T}\right)^{-1} p_{s_{1}} \times p_{s_{2}}\right\rangle\right|} d s_{1} d s_{2},
$$

where $p_{s_{i}}:=\frac{\partial p}{\partial s_{i}}$.

Proof. Since $F_{t_{0}}^{t_{0}+T} \circ p$ is a parameterization of $\mathcal{M}\left(t_{0}+T\right)$, the vectors $\nabla F_{t_{0}}^{t_{0}+T} p_{s_{i}}$ are tangent to $F_{t_{0}}^{t_{0}+T}(U)$. The advected surface area, by definition, is then

$$
S\left(F_{t_{0}}^{t_{0}+T}(U)\right)=\int_{U} \sqrt{\left|\left\langle\nabla F_{t_{0}}^{t_{0}+T} p_{s_{1}} \times \nabla F_{t_{0}}^{t_{0}+T} p_{s_{2}}, \nabla F_{t_{0}}^{t_{0}+T} p_{s_{1}} \times \nabla F_{t_{0}}^{t_{0}+T} p_{s_{2}}\right\rangle\right|} d s_{1} d s_{2} .
$$

This implies formula B.1 based on the general identity $M v \times M u=(\operatorname{det} M) M^{-T} v \times u$, which holds for any invertible square matrix $M$ and vectors $u$ and $v$.

Proposition 1. [Surface area of LCSs] Let $p\left(s_{1}, s_{2}\right)$ be a parameterization of a material surface $\mathcal{M}(t) \subset \mathbb{R}^{3}$ over the time interval $\left[t_{0}, t_{0}+T\right]$.

(i) Suppose that $\mathcal{M}(t)$ is a repelling hyperbolic LCS. Then we have

$$
S\left(F_{t_{0}}^{t_{0}+T}(U)\right)=\int_{U}\left|\operatorname{det}\left(\nabla F_{t_{0}}^{t_{0}+T}\right)\right| \cdot\left\|p_{s_{1}} \times p_{s_{2}}\right\| \frac{1}{\sqrt{\lambda_{3}}} d s_{1} d s_{2} .
$$

(ii) Suppose that $\mathcal{M}(t)$ is an attracting hyperbolic LCS. Then we have

$$
S\left(F_{t_{0}}^{t_{0}+T}(U)\right)=\int_{U}\left|\operatorname{det}\left(\nabla F_{t_{0}}^{t_{0}+T}\right)\right| \cdot\left\|p_{s_{1}} \times p_{s_{2}}\right\| \frac{1}{\sqrt{\lambda_{1}}} d s_{1} d s_{2} .
$$

(iii) Suppose that $\mathcal{M}(t)$ is a shear LCS. Then we have

$$
S\left(F_{t_{0}}^{t_{0}+T}(U)\right)=\int_{U}\left|\operatorname{det}\left(\nabla F_{t_{0}}^{t_{0}+T}\right)\right| \cdot\left\|p_{s_{1}} \times p_{s_{2}}\right\| \frac{1}{\sqrt[4]{\lambda_{1} \lambda_{3}}} d s_{1} d s_{2} .
$$

In the special case of a volume-preserving flow, we have

$$
S\left(F_{t_{0}}^{t_{0}+T}(U)\right)=\int_{U}\left\|p_{s_{1}} \times p_{s_{2}}\right\| \sqrt[4]{\lambda_{2}} d s_{1} d s_{2} .
$$

Proof. We proof the result for (iii), as cases (i) and (ii) are similar. The shear vector field $n_{ \pm}$has unit length, and hence $\left\langle p_{s_{1}} \times p_{s_{2}}, p_{s_{1}} \times p_{s_{2}}\right\rangle=\left\|p_{s_{1}} \times p_{s_{2}}\right\|^{2}$. Observe that

$$
\begin{aligned}
& S\left(F_{t_{0}}^{t_{0}+T}(U)\right)=\int_{U}\left|\operatorname{det}\left(\nabla F_{t_{0}}^{t_{0}+T}\right)\right| \cdot \sqrt{\left|\left\langle p_{s_{1}} \times p_{s_{2}},\left(C_{t_{0}}^{t_{0}+T}\right)^{-1} p_{s_{1}} \times p_{s_{2}}\right\rangle\right|} d s_{1} d s_{2} \\
& =\int_{U}\left|\operatorname{det}\left(\nabla F_{t_{0}}^{t_{0}+T}\right)\right| \cdot \| p_{s_{1}} \times p_{s_{2}} \mid \sqrt{\left|\left\langle n_{ \pm},\left(C_{t_{0}}^{t_{0}+T}\right)^{-1} n_{ \pm}\right\rangle\right|} d s_{1} d s_{2} .
\end{aligned}
$$




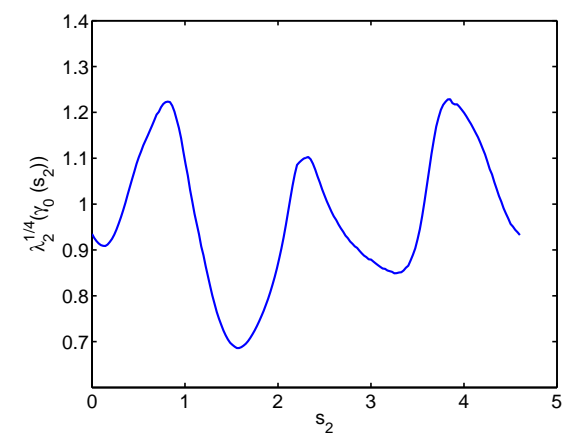

Figure B.15: $\sqrt[4]{\lambda_{2}}$ computed over a closed reduced shearline $\gamma_{0}$ for the steady ABC flow, obtained from the flow map $F_{0}^{150}$.

Using the definition of $n_{ \pm}$, one sees that $\left\langle n_{ \pm},\left(C_{t_{0}}^{t_{0}+T}\right)^{-1} n_{ \pm}\right\rangle=\frac{1}{\sqrt{\lambda_{1} \lambda_{3}}}$. Substituting this identity into (B.2) proves (iii) of Proposition 1. We can deduce the result for repelling and attracting LCSs similarly, using the fact that $\left\langle\xi_{3},\left(C_{t_{0}}^{t_{0}+T}\right)^{-1} \xi_{3}\right\rangle=\frac{1}{\lambda_{3}}$ for a repelling LCS, and $\left\langle\xi_{1},\left(C_{t_{0}}^{t_{0}+T}\right)^{-1} \xi_{1}\right\rangle=$ $\frac{1}{\lambda_{1}}$ for an attracting LCS.

Proposition 1 shows that the final surface area along a shear LCS in incompressible flow is obtained by integrating the initial surface element $\left\|p_{s_{1}} \times p_{s_{2}}\right\|$ weighted by $\sqrt[4]{\lambda_{2}}$. In Appendix A, we showed that $\lambda_{2}=1$ golds globally in space and time, and hence the corresponding shear LCS surface area is conserved. For the steady ABC flow, we find that $\lambda_{2}$ computed over elliptic LCSs oscillates around one. Fig. Appendix B shows this along a specific closed reduced shearline.

\section{Appendix C. Proof of Theorem 1}

We first need the following proposition which allows us to compute the normal repulsion and tangential shear in terms of $C_{t_{0}}^{t_{0}+T}$ and $n_{0}$.

Proposition 2. The quantities $\rho$ and $\sigma$ can be expressed as

$$
\begin{aligned}
\rho\left(x_{0}, n_{0}\right) & =\frac{1}{\sqrt{\left\langle n_{0},\left[C_{t_{0}}^{t_{0}+T}\left(x_{0}\right)\right]^{-1} n_{0}\right\rangle}}, \\
\sigma\left(x_{0}, n_{0}\right) & =\sqrt{\left\langle n_{0}, C_{t_{0}}^{t_{0}+T}\left(x_{0}\right) n_{0}\right\rangle-\frac{1}{\left\langle n_{0},\left[C_{t_{0}}^{t_{0}+T}\left(x_{0}\right)\right]^{-1} n\right\rangle}} .
\end{aligned}
$$


Proof. The proof of the first identity can be found in [15]. As for the second identity, we make use of the following formula for the unit normal of a multi-dimensional material surface [15]:

$$
n_{t}=\frac{\left[\left(\nabla F_{t}^{t_{0}}\right)^{*} n_{0}\right]}{\left|\left(\nabla F_{t}^{t_{0}}\right)^{*} n_{0}\right|} .
$$

We then obtain

$$
\begin{aligned}
{\left[\sigma\left(x_{0}, n_{0}\right)\right]^{2}=} & \left|\nabla F_{t_{0}}^{t_{0}+T}\left(x_{0}\right) n_{0}-\left\langle n_{t}, \nabla F_{t_{0}}^{t_{0}+T}\left(x_{0}\right) n_{0}\right\rangle n_{t}\right| \\
= & \left\langle n_{0},\left(\nabla F_{t_{0}}^{t_{0}+T}\left(x_{0}\right)\right)^{*} \nabla F_{t_{0}}^{t_{0}+T}\left(x_{0}\right) n_{0}\right\rangle-2\left\langle\nabla F_{t_{0}}^{t_{0}+T}\left(x_{0}\right) n_{0},\left\langle n_{t}, \nabla F_{t_{0}}^{t_{0}+T}\left(x_{0}\right) n_{0}\right\rangle n_{t}\right\rangle \\
& +\left\langle\left\langle n_{t}, \nabla F_{t_{0}}^{t_{0}+T}\left(x_{0}\right) n_{0}\right\rangle n_{t},\left\langle n_{t}, \nabla F_{t_{0}}^{t_{0}+T}\left(x_{0}\right) n_{0}\right\rangle n_{t}\right\rangle \\
= & \left\langle n_{0}, C_{t_{0}}^{t_{0}+T}\left(x_{0}\right) n_{0}\right\rangle-\left[\rho\left(x_{0}, n_{0}\right)\right]^{2} \\
= & \left\langle n_{0}, C_{t_{0}}^{t_{0}+T}\left(x_{0}\right) n_{0}\right\rangle-\frac{1}{\left\langle n_{0},\left[C_{t_{0}}^{t_{0}+T}\left(x_{0}\right)\right]^{-1} n_{0}\right\rangle},
\end{aligned}
$$

which proves the proposition.

We are now ready to prove Theorem 1 . Let $S$ be a hyperbolic LCS and $x_{0}$ a point on $S$. Thus, by definition, for any other surface $\tilde{S}$ passing through $x_{0}$ such that $T_{x_{0}} \tilde{S} \neq T_{x_{0}} S$, the normal repulsion rate $\rho\left(x_{0}, n_{0}^{\tilde{S}}\right)$ along $\tilde{S}$ at $x_{0}$ is smaller than the normal repulsion rate $\rho\left(x_{0}, n_{0}^{S}\right)$ along $S$ at $x_{0}$. Thus, at each point $x_{0}$ on $S$, the quantity $\rho\left(x_{0}, n_{0}\right)$ is maximized with respect to changes in $n_{0}$. Thus, we want to solve the following constrained optimization problem: maximize $\rho\left(x_{0}, n_{0}\right)$ with respect to $n_{0}$ under the constraint that $\left\|n_{0}\right\|=1$. To this end, expand $n_{0}$ in the eigen-basis $\left\{\xi_{1}, \xi_{2}, \xi_{3}\right\}$ of the Cauchy-Green strain tensor $C_{t_{0}}^{t_{0}+T}\left(x_{0}\right)$ :

$$
n_{0}=\sum_{i=1}^{3} n_{i} \xi_{i}
$$

We then have

$$
\rho\left(x_{0}, n_{0}\right)=\left(\sum_{i=1}^{3} \frac{n_{i}^{2}}{\lambda_{i}}\right)^{-1},
$$

and setting the gradient of $\rho\left(x_{0}, n_{0}\right)$ proportional to the gradient of the constraint $\left\|n_{0}\right\|=1$ yields

$$
\begin{aligned}
& -2 \rho^{2} \lambda_{2} \lambda_{3} n_{1}=\gamma n_{1}, \\
& -2 \rho^{2} \lambda_{1} \lambda_{3} n_{2}=\gamma n_{2} . \\
& -2 \rho^{2} \lambda_{1} \lambda_{2} n_{n}=\gamma n_{3},
\end{aligned}
$$

where $\gamma$ is an appropriate constant. Thus, assuming that $\lambda_{1}>0$, it follows that two of the coordinates $n_{1}, n_{2}, n_{3}$ must be zero. Therefore, the only extremum directions of the normal repulsion rate are the eigenvectors $\xi_{i}$ of the Cauchy-Green strain tensor. Since $\rho\left(x_{0}, \xi_{i}\right)=\sqrt{\lambda_{i}}$, it follows that $n_{0}=\xi_{3}$ is the global maximum of the normal repulsion $\rho$ with respect to changes in $n_{0}$. Therefore, a repelling hyperbolic LCS is necessarily orthogonal to $\xi_{3}$. The proof of statement $(i i)$ is analogous, but involves the global minimum of the normal repulsion rate.

The prove statement (iii), we need to find the maximizing normal directions $n_{0}$ of the tangential shear $\sigma\left(x_{0}, n_{0}\right)$ under the constraint that $\left\|n_{0}\right\|=1$. We again represent $n_{0}$ in the eigen-basis $\left\{\xi_{1}, \xi_{2}, \xi_{3}\right\}$ of $C_{t_{0}}^{t_{0}+T}\left(x_{0}\right)$ as

$$
n_{0}=\sum_{i=1}^{3} n_{i} \xi_{i}, \quad \sum_{i=1}^{3} n_{i}^{2}=1,
$$


and seek to maximize $\sigma\left(n_{1}, n_{2}, n_{3}\right):=\sigma\left(x_{0}, n_{0}\right)$ subject to the constraint $\sum n_{i}^{2}=1$. Note that

$$
\sigma\left(n_{1}, n_{2}, n_{3}\right)=\sqrt{\sum \lambda_{i} n_{i}^{2}-\frac{1}{\sum \lambda_{i}^{-1} n_{i}^{2}}} .
$$

Setting the gradient of $\sigma\left(n_{1}, n_{2}, n_{3}\right)$ proportional to the gradient of the constraint $\sum n_{i}^{2}=1$ leads to the equations

$$
\frac{1}{2 \sigma}\left(2 n_{i} \lambda_{i}+\frac{2 n_{i}}{\lambda_{i}\left(\sum \lambda_{i}^{-1} n_{i}^{2}\right)^{2}}\right)=2 \gamma n_{i},
$$

which can also be rewritten as

$$
\frac{1}{2 \sigma}\left(2 n_{i} \lambda_{i}+\frac{2 n_{i}}{\lambda_{i}\left(\sum \lambda_{i}^{-1} n_{i}^{2}\right)^{2}}\right)=2 \gamma n_{i},
$$

or, equivalently,

$$
n_{i}\left[\left(\sum_{j=1}^{3} \frac{n_{j}^{2}}{\lambda_{j}}\right)^{2} \lambda_{i}+\frac{1}{\lambda_{i}}-2 \sigma \gamma\right]=0, \quad i=1,2,3 .
$$

Assume now that for some index $i$, we have $n_{i} \neq 0$, i.e., the $i^{\text {th }}$ component of the unknown normal vector $n_{0}$ of the shear LCS is nonzero. In that case, we must have

$$
\frac{2 \sigma \gamma-\frac{1}{\lambda_{i}}}{\lambda_{i}}=K \stackrel{\text { def. }}{=}\left(\sum_{j=1}^{3} \frac{n_{j}^{2}}{\lambda_{j}}\right)^{2}
$$

where the constant $K>0$ is the same for any choice of $i$. Taking the square of equation (C.6) gives

$$
\lambda_{i}^{2}-\frac{2 \sigma \gamma}{K} \lambda_{i}+\frac{1}{K}=0
$$

and hence there can be at most two distinct $\lambda_{i}$ values for which (C.6) holds. Also note that there has to be at least two distinct $\lambda_{i}$ values for which (C.6) holds, otherwise $n_{0}$ would be an eigenvector, and hence a local minimizer of the tangential shear. We conclude that there exist precisely two eigenvalues, $\lambda_{k}$ and $\lambda_{l}>\lambda_{k}$, of $C_{t_{0}}^{t_{0}+T}$ that satisfy (C.6).

All eigenvalues of $C_{t_{0}}^{t_{0}+T}\left(x_{0}\right)$ are simple by assumption. Therefore, by our argument above, $n_{0}$ must be of the form

$$
n_{0}=n_{k} \xi_{k}+n_{l} \xi_{l}, \quad n_{k}^{2}+n_{l}^{2}=1
$$

Substituting the expression (C.7) into (C.6) with $i=k$ and $i=l$, eliminating the common constant $2 \sigma \gamma$ from the resulting two equations, and using the notation

$$
a^{2}=n_{k}^{2}, \quad b^{2}=n_{l}^{2},
$$

we obtain the system of equations

$$
\begin{aligned}
\lambda_{k}\left(\frac{a^{2}}{\lambda_{k}}+\frac{b^{2}}{\lambda_{l}}\right)^{2}+\frac{1}{\lambda_{k}} & =\lambda_{l}\left(\frac{a^{2}}{\lambda_{k}}+\frac{b^{2}}{\lambda_{l}}\right)^{2}+\frac{1}{\lambda_{l}}, \\
a^{2}+b^{2} & =1,
\end{aligned}
$$

for the unknowns $a^{2}$ and $b^{2}$. The solution of these equations is given by

$$
a^{2}=\frac{\sqrt{\lambda_{k}}}{\sqrt{\lambda_{k}}+\sqrt{\lambda_{l}}}, \quad b^{2}=\frac{\sqrt{\lambda_{l}}}{\sqrt{\lambda_{k}}+\sqrt{\lambda_{l}}}
$$


Thus $n_{0}$ must take the more specific form

$$
\begin{aligned}
n_{0} & =a \xi_{k}+b \xi_{l}, \quad \lambda_{k}<\lambda_{l}, \\
a^{2}=\frac{\sqrt{\lambda_{k}}}{\sqrt{\lambda_{k}}+\sqrt{\lambda_{l}}}, & b^{2}=\frac{\sqrt{\lambda_{l}}}{\sqrt{\lambda_{k}}+\sqrt{\lambda_{l}}},
\end{aligned}
$$

for some choice of $k, l \in\{1,2,3\}$. We now check which of these extrema are indeed local maxima. Computing the tangential shear for expressions (C.10) yields

$$
\begin{aligned}
\sigma\left(x_{0}, n_{0}\right) & =\sqrt{\left\langle n_{0}, C_{t_{0}}^{t_{0}+T} n_{0}\right\rangle-\frac{1}{\left\langle n_{0},\left[C_{t_{0}}^{t_{0}+T}\right]^{-1} n_{0}\right\rangle}} \\
& =\sqrt{a^{2} \lambda_{k}+b^{2} \lambda_{l}-\frac{\lambda_{k} \lambda_{l}}{a^{2} \lambda_{l}+b^{2} \lambda_{k}}} \\
& =\left|\sqrt{\lambda_{l}}-\sqrt{\lambda_{k}}\right| .
\end{aligned}
$$

Next we prove that $k=1$ and $l=3$ must hold for the normal $n_{0}$ in formula (C.10). Assume the contrary, i.e., assume that the pair of eigenvalues $\left(\lambda_{k}, \lambda_{l}\right)$ in formula (C.10) does not coincide with the pair $\left(\lambda_{1}, \lambda_{2}\right)$. We only consider the case of $\lambda_{k} \neq \lambda_{1}$, because the case of $\lambda_{l} \neq \lambda_{3}$ can be handled in an identical fashion. Assuming $\lambda_{k} \neq \lambda_{1}$, define the unit normal

$$
\hat{n}_{0}=\sqrt{\frac{\sqrt{\lambda_{1}}}{\sqrt{\lambda_{1}}+\sqrt{\lambda_{3}}}} \xi_{1}+\sqrt{\frac{\sqrt{\lambda_{3}}}{\sqrt{\lambda_{1}}+\sqrt{\lambda_{3}}}} \xi_{3} .
$$

Note that $\hat{n}_{0} \neq n_{0}$ by our assumption, and by formula (C.11), we have

$$
\sigma\left(x_{0}, n_{0}\left(x_{0}\right)\right)=\left|\sqrt{\lambda_{l}}-\sqrt{\lambda_{k}}\right|<\left|\sqrt{\lambda_{n}}-\sqrt{\lambda_{1}}\right|=\sigma\left(x_{0}, \hat{n}_{0}\right),
$$

which contradicts our maximality assumption for shear LCS in Definition 1 (namely that a shear LCS has tangential shear no less than any perturbations of its normal direction $n_{0}$ ).

We have, therefore, obtained that for any shear LCS, the normal vector $n_{0}\left(x_{0}\right)$ featured in (C.10) must necessarily be of the more specific form

$$
n_{0}= \pm \sqrt{\frac{\sqrt{\lambda_{1}}}{\sqrt{\lambda_{1}}+\sqrt{\lambda_{3}}}} \xi_{1} \pm \sqrt{\frac{\sqrt{\lambda_{3}}}{\sqrt{\lambda_{1}}+\sqrt{\lambda_{3}}}} \xi_{3}
$$

where $\lambda_{1}$ and $\lambda_{n}$ are multiplicity-one eigenvalues of the Cauchy-Green strain tensor $C_{t_{0}}^{t_{0}+T}\left(x_{0}\right)$, and the two \pm signs can be chosen independently of one another. All in all, formula (C.12) defines two linearly independent unit normal directions, corresponding to maximal positive and maximal negative shear. This proves that a shear LCS is necessarily orthogonal to either $n_{+}$or $n_{-}$.

\section{Appendix D. Proof of Theorem 2 and relation to Frobenius Integrability}

\section{Appendix D.1. Proof of Theorem 2}

For a general three-dimensional vector field $v$, consider the problem of finding a surface $S$ orthogonal to $v$. The following proposition shows that a necessary condition for the existence of $S$ is that the helicity of $v$

$$
H_{v}(x)=\langle\nabla \times v, v\rangle
$$

must vanish on $S$. This fact was pointed out in [20]; here we provide an alternative proof using Stokes' Theorem. 
Proposition 3. Let $v$ be a smooth vector field in $\mathbb{R}^{3}$ and $S$ a surface orthogonal to $v$. Then for any $x \in S$, the helicity of vmust vanish, i.e.,

$$
H_{v}(x):=\langle\nabla \times v(x), v(x)\rangle=0 .
$$

Proof. Consider an open neighborhood $D \subset S$ of $x$ in $S$. By Stokes' Theorem, we have that

$$
\int_{D}(\nabla \times v) \cdot n=\int_{C} v \cdot d r
$$

Since $v$ is orthogonal to $S$, the integral on the right-hand side of (D.3) is zero. Thus, since $v=$ $\langle v, n\rangle n$, we have

$$
\int_{D} \frac{1}{\langle v, n\rangle} H_{v} d A=0
$$

Since $D$ was arbitrary, $H_{v}$ must vanish on $S$.

Theorem 2 then follows directly from Theorem 1 and Proposition 3.

Appendix D.2. Relation to Frobenius Integrability

We can rephrase the problem of computing a surface orthogonal to $\xi_{3}$ for repelling hyperbolic, $\xi_{1}$ for attracting hyperbolic, and $n_{ \pm}$for shear LCS as finding surfaces tangent to $\left\{\xi_{1}, \xi_{2}\right\},\left\{\xi_{2}, \xi_{3}\right\}$ and $\left\{\xi_{2}, n_{ \pm} \times \xi_{2}\right\}$, respectively. The problem of finding surfaces tangent to two specified vector fields is then related to the Frobenius Integrability Theorem [1].

As a special case, this theorem states that if $X$ and $Y$ are two vector fields in $\mathbb{R}^{3}$, then necessary and sufficient conditions for the existence of a foliation of $\mathbb{R}^{3}$ by surfaces tangent $X$ and $Y$ is that

$$
[X, Y] \in \operatorname{Span}\{X, Y\}
$$

In our context, we do not seek to find global foliations of $\mathbb{R}^{3}$, but only individual, isolated surfaces. Nevertheless, as we show in this section, the Frobenius condition (D.5) is still a necessary, albeit not sufficient condition for the existence of such surfaces.

If $X, Y$ and $Z$ are smooth vector fields in $\mathbb{R}^{3}$, consider both the Frobenius and helicity conditions

$$
\begin{aligned}
& F_{X, Y, Z}=\langle[X, Y], Z\rangle=0, \\
& H_{V}\langle\nabla \times Z, Z\rangle=0 .
\end{aligned}
$$

We show in Proposition 4 that the zero sets of $F$ and $H_{Z}$ coincide, provided that $X, Y$ and $Z$ are pairwise orthogonal vector fields. Combined with Theorem 2, Proposition 4 then shows that (D.5) is a necessary condition. We make use of the following lemma, which states that the zero sets of $F$ and $H_{Z}$ are invariant under a nonlinear rescaling of the three vector fields involved.

Lemma 3. Let $X, Y$ and $Z$ be pairwise orthogonal vector fields in $\mathbb{R}^{3}$, and $\phi_{1}, \phi_{2}, \phi_{3}$ be nonzero scalar function on $\mathbb{R}^{3}$. Then we have that

$$
\begin{aligned}
& F_{\phi_{1} X, \phi_{2} Y, \phi_{3} Z}=\phi_{1} \phi_{2} \phi_{3} F_{X, Y, Z}, \\
& H_{\phi_{3} Z}=\phi_{3}^{2} H_{Z} .
\end{aligned}
$$

In particular, the zero sets of $F_{\phi_{1} X, \phi_{2} Y, \phi_{3} Z}$ and $H_{\phi_{3} Z}$ coincide with those of $F_{X, Y, Z}$ and $H_{Z}$, respectively. 
Proof. By definition, we have that

$$
\begin{aligned}
& F_{\phi_{1} X, \phi_{2} Y, \phi_{3} Z}=\left\langle\left[\phi_{1} X, \phi_{2} Y\right], \phi_{3} Z\right\rangle \\
& =\phi_{3}\left\langle D\left(\phi_{1} X\right) Y-D\left(\phi_{2} Y\right) X, Z\right\rangle=\phi_{3}\left\langle\left(\nabla \phi_{1}\right) X^{T} Y+\phi_{1} \phi_{2} D X Y-\phi_{1}\left(\nabla \phi_{2}\right) Y^{T} X-\phi_{1} \phi_{2} D Y X, Z\right\rangle .
\end{aligned}
$$

However, $X^{T} Y=Y^{T} X=0$ by our orthogonality assumption, and hence

$$
F_{\phi_{1} X, \phi_{2} Y, \phi_{3} Z}=\phi_{1} \phi_{2} \phi_{3}\langle[X, Y], Z\rangle=\phi_{1} \phi_{2} \phi_{3} F_{X, Y, Z}
$$

As for the claim on the helicity, note that

$$
\begin{aligned}
& H_{\phi_{3} Z}=\left\langle\nabla \times\left(\phi_{3} Z\right), \phi_{3} Z\right\rangle \\
& =\phi_{3}\left\langle\nabla \phi_{3} \times Z+\phi_{3}(\nabla \times Z), Z\right\rangle=\phi_{3}^{2}\langle\nabla \times Z, Z\rangle
\end{aligned}
$$

Proposition 4. Let $X, Y$ and $Z$ be a smoothly varying, pairwise orthogonal family of vector fields in $\mathbb{R}^{3}$. Then the zero set of $F=\langle[X, Y], Z\rangle$ coincides with the zero set of $H_{Z}\langle\nabla \times Z, Z\rangle=0$.

Proof. By Lemma 3, it suffices to assume that $X, Y$ and $Z$ is an orthonormal family of vector fields. Assume that

$$
\langle[X, Y], Z\rangle=\langle(\nabla X) Y-(\nabla Y) X, Z\rangle=0 .
$$

Then differentiating the orthonormality assumptions $\langle X, Y\rangle=\langle X, Z\rangle=0,\|X\|=\|Y\|=\|Z\|=1$, we obtain

$$
\begin{aligned}
& (\nabla X)^{T} Z+(\nabla Z)^{T} X=0, \\
& (\nabla Y)^{T} Z+(\nabla Z)^{T} Y=0,
\end{aligned}
$$

which, after substitution into the Frobenius relation (D.11), yields

$$
\left\langle X,\left[\nabla Z-\nabla Z^{T}\right] Y\right\rangle=0
$$

Now we recall the following general identity for vector fields in $\mathbb{R}^{3}$ :

$$
\left[\nabla a-\nabla a^{T}\right] b=(\nabla \times a) \times b .
$$

Applying this to $a=Z$ and $b=Y$, we obtain

$$
\langle X,(\nabla \times Z) \times Y\rangle=0 .
$$

Finally, using the identity $(a \times b) \cdot c=(b \times c) \cdot a$ with $a=\nabla \times Z, b=Y$, and $c=X$, we obtain that (D.11) is equivalent to

$$
\langle\nabla \times Z, Z\rangle=0
$$

as claimed.

Remark 8. Proposition 4 shows that the helicity conditions in Theorem 2 are equivalent to the Frobenius conditions $\left\langle\left[\xi_{1}, \xi_{2}\right], \xi_{3}\right\rangle=0,\left\langle\left[\xi_{2}, \xi_{3}\right], \xi_{1}\right\rangle=0$, and $\left\langle\left[\xi_{2}, n_{ \pm} \times \xi_{2}\right], n_{ \pm}\right\rangle=0$ for repelling hyperbolic, attracting hyperbolic, and shear LCSs, respectively.

Remark 9. Frobenius Integrability Theorem applied to the existence of tangent foliations provides a necessary and sufficient condition. By contrast, the zero helicity condition (and its equivalent Frobenius condition) are only necessary conditions for the existence of isolated surfaces normal to a vector field $v$. For example, let $v(x, y, z)=(y, z, x)$. Then $H_{v}(x, y, z)=\langle\nabla \times v, v\rangle=-y-z-x$, which has a plane as its zero set, but this plane is not orthogonal to $v$. Thus $H_{v}(x, y, z)=0$ is not sufficient for the existence of a surface orthogonal to $v$. 


\section{Appendix E. Proof of Theorem 3}

At any point $x_{0} \in \Pi\left(s_{1}\right)$, a tangent vector to such a potential intersection curve $\gamma_{s_{1}}=\Pi\left(s_{1}\right) \cap$ $\mathcal{M}\left(t_{0}\right)$ must be orthogonal both to the unit normal vector $n_{\Pi\left(s_{1}\right)}$ of $\Pi\left(s_{1}\right)$, and either to $\xi_{3}$ (repelling hyperbolic barriers), to $\xi_{1}$ (attracting hyperbolic barriers) or to $n_{ \pm}$(shear barriers). As a result, the intersection of a transport barrier $\mathcal{M}\left(t_{0}\right)$ with $\Pi\left(s_{1}\right)$ must be a curve tangent to one of the following three vector fields on $\Pi\left(s_{1}\right)$ :

$$
\begin{gathered}
u_{\xi_{3}}\left(x_{0} ; s_{1}\right)=n_{\Pi\left(s_{1}\right)}\left(x_{0}\right) \times \xi_{3}\left(x_{0}\right), \quad u_{\xi_{1}}\left(x_{0} ; s_{1}\right)=n_{\Pi\left(s_{1}\right)}\left(x_{0}\right) \times \xi_{1}\left(x_{0}\right), \\
u_{n_{ \pm}}\left(x_{0} ; s_{1}\right)=n_{\Pi\left(s_{1}\right)} \times n_{ \pm}\left(x_{0}\right) .
\end{gathered}
$$

We call $u_{\xi_{3}}\left(x_{0} ; s_{1}\right)$ the reduced strain vector field and $u_{\xi_{1}}\left(x_{0} ; s_{1}\right)$ the reduced stretch vector field on the reference surface $\Pi\left(s_{1}\right)$. Similarly, we call and $u_{n_{ \pm}}\left(x_{0} ; s_{1}\right)$ the reduced shear vector fields on $\Pi\left(s_{1}\right)$. Since the manifold family $\Pi\left(s_{1}\right)$ is assumed orientable, the unit normal vector field $n_{\Pi\left(s_{1}\right)}\left(x_{0}\right)$ can be selected smoothly globally on $\Pi\left(s_{1}\right)$. By contrast, the vector fields $\xi_{3}\left(x_{0}\right)$ and $n_{ \pm}\left(x_{0}\right)$ are typically not globally orientable, and can only be selected smoothly over open subset of $\Pi\left(s_{1}\right)$.

The resulting local orientability of the vector fields $u_{\xi_{3}}\left(x_{0} ; s_{1}\right)$ and $u_{n_{ \pm}}\left(x_{0} ; s_{1}\right)$ on $\Pi\left(s_{1}\right)$ is enough for the construction of all possible intersection curves $\gamma_{s_{1}}=\Pi\left(s_{1}\right) \cap \mathcal{M}\left(t_{0}\right)$. This can be achieved by solving piecewise oriented versions of one of the differential equations (14)-(18). Furthermore, any trajectory of these differential equations that represents a true intersection curve $\gamma_{s_{1}}=\Pi\left(s_{1}\right) \cap \mathcal{M}\left(t_{0}\right)$ with a barrier $\mathcal{M}\left(t_{0}\right)$ must necessarily lie in the zero set of the appropriate helicity function featured in Theorem 2.

\section{Appendix F. Numerical aspects of computing hyperbolic and shear LCS}

\section{Appendix F.1. Computing hyperbolic LCS}

The algorithmic steps H1-H6 provide a way to compute intersections between hyperbolic LCSs and a family of reference planes. This involves computing trajectories of the reduced strain vector fields in (14) and (16), both of which will generally have singularities and orientational discontinuities. A systematic description of the numerical procedures to deal with these issues can be found in [7].

\section{Appendix F.2. Computing shear LCS}

Again, the algorithmic steps SH1-SH5 provide a way to compute intersections of shear LCSs and with a family of reference planes. The reduced shear vector field (18) has singularities and orientational discontinuities that can be handles as in the case of reduced strain vector field.

An additional complication for shear LCS is the smooth choice of $n_{ \pm}$along reduced shear trajectories. The normal fields $n_{ \pm}$have the general form

$$
n_{ \pm}=\alpha \xi_{1} \pm \beta \xi_{3},
$$

representing four different directions in the three-dimensional phase space.

In the theory of transport barriers for 2D flows in [16], an analogous shear vector field

$$
\eta_{ \pm}=\alpha \xi_{1} \pm \beta \xi_{2}
$$

arises. For this field, one can adopt the global orientation convention $\xi_{2}=\Omega \xi_{1}$, where $\Omega$ denotes the operator of counter-clockwise rotation by 90 degrees. With this way of computing $\xi_{2}$ in terms of $\xi_{1}$, the vector field $\eta_{ \pm}$only represents two vectors due to the sign ambiguity of $\xi_{1}$, which we assume $\xi_{2}$ inherits. One can then solve for trajectories of $\eta_{ \pm}$by solving

$$
r^{\prime}(s)=\operatorname{sign}\left(\left\langle\eta_{ \pm}(r(s)), r(s-\Delta)\right\rangle\right) \eta_{ \pm}(r(s)),
$$

with $\Delta$ denoting a numerical time step. 
In the 3D setting of the present paper, we cannot find a linear transformation $\Omega$ that would globally fix the orientation of $\xi_{3}$ relative to $\xi_{1}$. One therefore does not have simply two globally defined vector fields to integrate. Rather, one starts the integration and has to consider at each point along a reduced shearline all four possible directions, resulting in four possible choices of $n_{0}$ at that point. The correct vector to select is the one that results in a smooth reduced shearline (as the transverse intersection of a shear LCS with the reference plane). The method used in this paper to select the correct vectors at each time step is:

1) At the initial condition $r(0)$ in the reference plane $\Pi\left(s_{1}\right)$, compute the vectors $\xi_{1}(r(0))$ and $\xi_{3}(r(0))$.

2) Assuming one has the solution $r\left(s_{2}\right)$ and the vectors $\xi_{1}\left(r\left(s_{2}\right)\right) \xi_{3}\left(r\left(s_{2}\right)\right)$ stored, one computes the reduced shearline at the point $r\left(s_{2}+\Delta\right)$ by matching the direction of $\xi_{1}\left(r\left(s_{2}+\Delta\right)\right)$ and $\xi_{3}\left(r\left(s_{2}+\right.\right.$ $\Delta)$ ) with the direction of $\xi_{1}\left(r\left(s_{2}\right)\right)$ and $\xi_{3}\left(r\left(s_{2}\right)\right)$ when forming $n_{ \pm}\left(r\left(s_{2}\right)+\Delta\right)$.

\section{References}

[1] R. Abraham, J. E. Marsden, and T. Ratiu. Manifolds, tensor analysis, and applications, volume 75 of Applied Mathematical Sciences. Springer-Verlag, New York, second edition, 1988. ISBN 0-387-96790-7. doi: 10.1007/978-1-4612-1029-0. URL http://dx.doi.org/10.1007/978-1-4612-1029-0.

[2] C. Basdevant and T. Philipovitch. On the validity of the weiss criterion in two-dimensional turbulence. Physica D: Nonlinear Phenomena, 73: 17 - 30, 1994. ISSN 0167-2789. doi: 10.1016/0167-2789(94)90222-4. URL http://www.sciencedirect.com/science/article/pii/0167278994902224.

[3] J.H. Bettencourt, C. Lopez, and E. Hernandez-Garcia. Characterization of coherent structures in three-dimensional turbulent flows using the finite-size lyapunov exponent. Journal of Physics A: Mathematical and Theoretical, 46(25):254022, 2013. URL http://stacks . iop.org/1751-8121/46/i=25/a=254022.

[4] G. Boffetta, G. Lacorata, G. Redaelli, and A. Vulpiani. Detecting barriers to transport: a review of different techniques. Physica D: Nonlinear Phenomena, 159: 58 - 70, 2001. ISSN 0167-2789. doi: 10.1016/S0167-2789(01)00330-X. URL http://www.sciencedirect.com/science/article/pii/S016727890100330X.

[5] M. Budisic and I. Mezic. Geometry of the ergodic quotient reveals coherent structures in flows. Physica D: Nonlinear Phenomena, 241(15):1255 - 1269, 2012. ISSN 0167-2789. doi: 10.1016/j.physd.2012.04.006. URL http://www.sciencedirect.com/science/article/pii/S0167278912001108.

[6] M. J. Olascoaga J. G. Goni F. J. Beron-Vera, Y. Wang and G. Haller. Objective detection of oceanic eddies and the agulhas leakage. Journal of Physical Oceanography. In press, 2013.

[7] M. Farazmand and G. Haller. Computing lagrangian coherent structures from their variational theory. Chaos: An Interdisciplinary Journal of Nonlinear Science, 22(1):013128, 2012. doi: 10.1063/1.3690153. URL http://link.aip.org/link/?CHA/22/013128/1.

[8] M. Farazmand and G. Haller. Erratum and addendum to "A variational theory of hyperbolic lagrangian coherent structures" [physica d 240 (2011) 574. Physica D: Nonlinear Phenomena, 241(4):439 - 441, 2012. ISSN 0167-2789. doi: http://dx.doi.org/10.1016/j.physd.2011.09.013. URL http://www.sciencedirect.com/science/article/pii/S0167278911002600.

[9] M. Farazmand and G. Haller. Attracting and repelling lagrangian coherent structures from a single computation. Chaos: An Interdisciplinary Journal of Nonlinear Science, 23(2):023101, 2013. doi: 10.1063/1.4800210. URL http://link.aip.org/link/?CHA/23/023101/1. 
[10] G. Froyland. An analytic framework for identifying finite-time coherent sets in time-dependent dynamical systems. Physica D: Nonlinear Phenomena, 250 (0):1 - 19, 2013. ISSN 0167-2789. doi: 10.1016/j.physd.2013.01.013. URL http://www.sciencedirect.com/science/article/pii/S0167278913000286.

[11] M. A. Green, C. W. Rowley, and G. Haller. Detection of lagrangian coherent structures in threedimensional turbulence. Journal of Fluid Mechanics, 572:111-120, 1 2007. ISSN 1469-7645. doi: $10.1017 /$ S0022112006003648.

[12] G. Haller H. Teramoto and T. Komatsuzaki. Detecting invariant manifolds as stationary lcss in autonomous dynamical systems. Chaos. submitted, 2013.

[13] G. Haller. Distinguished material surfaces and coherent structures in threedimensional fluid flows. Physica D: Nonlinear Phenomena, 149(4):248 277, 2001. ISSN 0167-2789. doi: 10.1016/S0167-2789(00)00199-8. URL http://www. sciencedirect.com/science/article/pii/S0167278900001998.

[14] G. Haller. An objective definition of a vortex. Journal of Fluid Mechanics, 525:1-26, 12005. ISSN 1469-7645. doi: 10.1017/S0022112004002526.

[15] G. Haller. A variational theory of hyperbolic lagrangian coherent structures. Physica D: Nonlinear Phenomena, 240(7):574 - 598, 2011. ISSN 0167-2789. doi: 10.1016/j.physd.2010.11.010. URL http://www.sciencedirect.com/science/article/pii/S0167278910003143.

[16] G. Haller and F. J. Beron-Vera. Geodesic theory of transport barriers in two-dimensional flows. Physica D: Nonlinear Phenomena, 241(20):1680 - 1702, 2012. ISSN 0167-2789. doi: 10.1016/j.physd.2012.06.012. URL http://www.sciencedirect.com/science/article/pii/S016727891200187X.

[17] G. Haller and F.J. Beron-Vera. Lagrangian vortices: The black holes of turbulence. preprint, 2013.

[18] J. Jeong and F. Hussain. On the identification of a vortex. Journal of Fluid Mechanics, 285: 69-94, 1 1995. ISSN 1469-7645. doi: 10.1017/S0022112095000462.

[19] D. Karrasch. Comment on "A variational theory of hyperbolic lagrangian coherent structures", physica d 240 (2011) 574. Physica D: Nonlinear Phenomena, 241(17):1470 1473, 2012. ISSN 0167-2789. doi: http://dx.doi.org/10.1016/j.physd.2012.05.008. URL http://www.sciencedirect.com/science/article/pii/S0167278912001443.

[20] K. L. Palmerius, M. Cooper, and A. Ynnerman. Flow field visualization using vector field perpendicular surfaces. In Spring Conference on Computer Graphics, 2009.

[21] F. Lekien, S. C. Shadden, and J. E. Marsden. Lagrangian coherent structures in n-dimensional systems. Journal of Mathematical Physics, 48(6):065404, 2007. doi: 10.1063/1.2740025. URL http://link. aip.org/link/?JMP/48/065404/1.

[22] J. D. Meiss. Symplectic maps, variational principles, and transport. Rev. Mod. Phys., 64:795-848, Jul 1992. doi: 10.1103/RevModPhys.64.795. URL http://link.aps.org/doi/10.1103/RevModPhys.64.795.

[23] T. Peacock and J. Dabiri. Introduction to focus issue: Lagrangian coherent structures. Chaos: An Interdisciplinary Journal of Nonlinear Science, 20(1):017501, 2010. doi: 10.1063/1.3278173. URL http://link.aip.org/link/?CHA/20/017501/1.

[24] D. Rosner. Transport Processes in Chemically Reacting Flow Systems. Dover Publications, 2000. 
[25] R. Schneider and R. Kleiber. Plasma Physics - Confinement, transport and collective effects, pages 425-441. Springer, Heidelberg, 2005.

[26] M. H. M. Sulman, H. S. Huntley, B. L. Lipphardt, and A. D. Kirwan. Out of Flatland: Three-Dimensional Aspects of Lagrangian Transport in Geophysical Fluids, pages 77-84. American Geophysical Union, 2012. ISBN 9781118704578. doi: 10.1029/2012GM001279. URL http://dx.doi.org/10.1029/2012GM001279.

[27] P. Tallapragada, S. D. Ross, and D. G. Schmale. Lagrangian coherent structures are associated with fluctuations in airborne microbial populations. Chaos: An Interdisciplinary Journal of Nonlinear Science, 21(3):033122, 2011. doi: 10.1063/1.3624930. URL http://link. aip.org/link/?CHA/21/033122/1.

[28] M. Toda. Geometrical Structures Of Phase Space In Multi-dimensional Chaos: Applications To Chemical Reaction Dynamics In Complex Systems. Advances in Chemical Physics Series. John Wiley \& Sons, 2005. ISBN 9780471711575. URL http://books . google.ch/books?id=1CQpAAAAYAAJ.

[29] J.B. Weiss. Transport and Mixing in Geophysical Flows, in: Lecture Notes in Physics, volume 744. Springer, New York, 2008. 